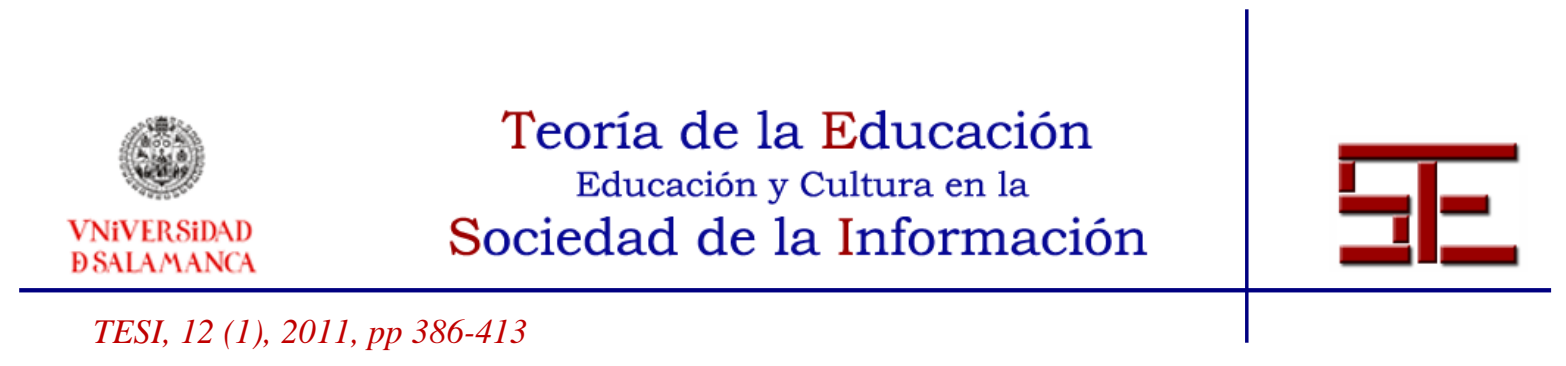

\title{
LA INTERDISCIPLINARIEDAD EN LA EDUCACIÓN SUPERIOR: PROPUESTA DE UNA GUÍA PARA EL DISEÑO DE JUEGOS DE ROL
}

Resumen: Con este artículo pretendemos configurar una metodología docente que se integra en las demandas y necesidades del Espacio Europeo de Educación Superior (EEES), en el que el alumno debe adquirir competencias profesionales que le garanticen la empleabilidad tras la finalización de sus estudios. Se propone un modelo de juego de rol de carácter interdisciplinar basado en la participación y colaboración de profesores de diferentes áreas de conocimiento y de alumnos de distintos títulos de grado. Este modelo ofrece al docente una guía que facilita el diseño y desarrollo de juegos de rol, cuyo objetivo es la adquisición y puesta en práctica de competencias genéricas y específicas de los nuevos planes de estudio involucrados. El uso de una metodología activa en nuestras aulas implica la dinamización de la docencia presencial -porque el juego es un elemento que invita a la participación y motivación del estudiante-, cumpliéndose con ello uno de nuestros objetivos principales: enseñar al alumno a aprender a aprender, simulando situaciones cuasireales en el contexto académico.

Palabras clave: educación superior; juegos de rol; innovación educativa; metodologías activas; interdisciplinariedad.

Deborah García Magna, Cristina Castillo Rodríguez, Sonia Rios

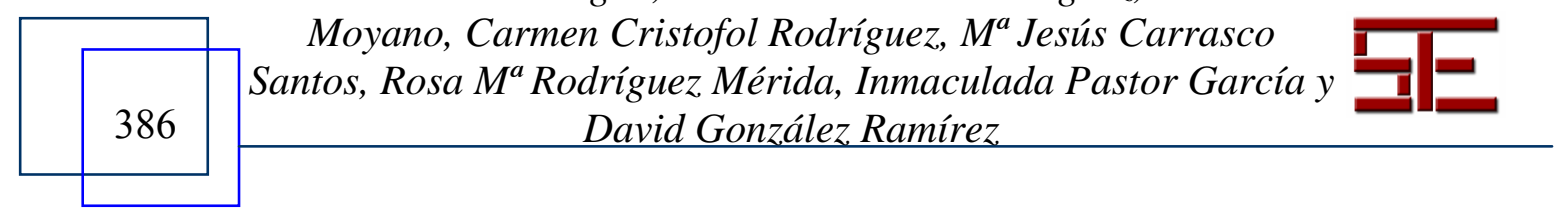




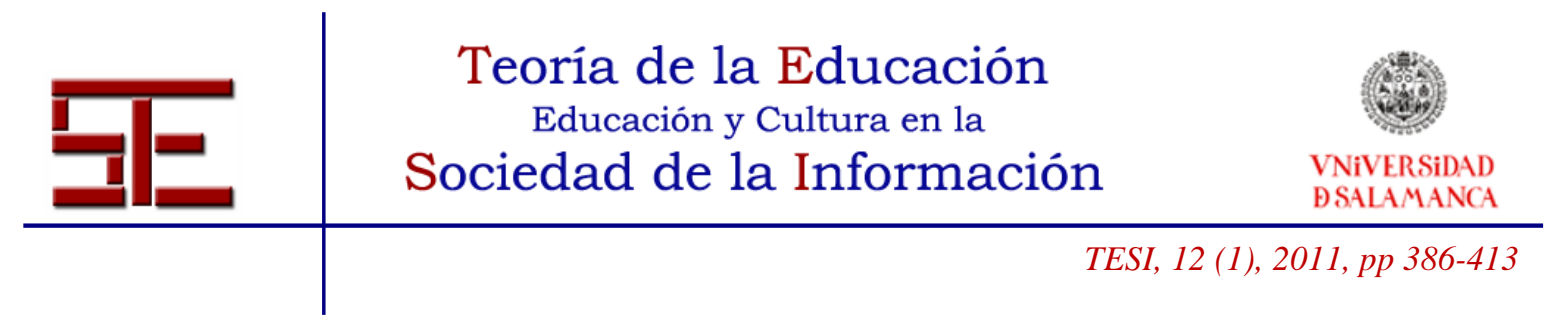

\title{
INTERDISCIPLINARIETY IN HIGHER EDUCATION: A GUIDE PROPOSAL FOR ROLE PLAY DESIGN
}

\begin{abstract}
The aim of this article is to establish a teaching methodology that is integrated into the requests and necessities of the European Higher Education Area (EHEA), in which the undergraduate student must acquire all the professional competences guaranteeing the employability after his/her studies. We propose an interdisciplinary role play model based on the participation and collaboration among university teachers from different knowledge fields as well as undergraduate students from a great variety of the new Bachelor degrees. This model offers the teacher a useful guide for the design and the development of role play activities, whose aim is the acquisition and the implementation of general and specific competences of the new curricula. The use of an active methodology in our classrooms implies the dinamization of the face to face teaching, since the game is the perfect element inviting the participation and motivation of the student. Thus, we fulfill one of our main objectives, that is, teaching the student to learn how to learn through the simulation of quasireal situations in the academic context.
\end{abstract}

Keywords: higher education; role play; teaching innovation; active methodologies; interdisciplinariety

Deborah García Magna, Cristina Castillo Rodríguez, Sonia

Rios Moyano, Carmen Cristofol Rodríguez, Ma Jesús

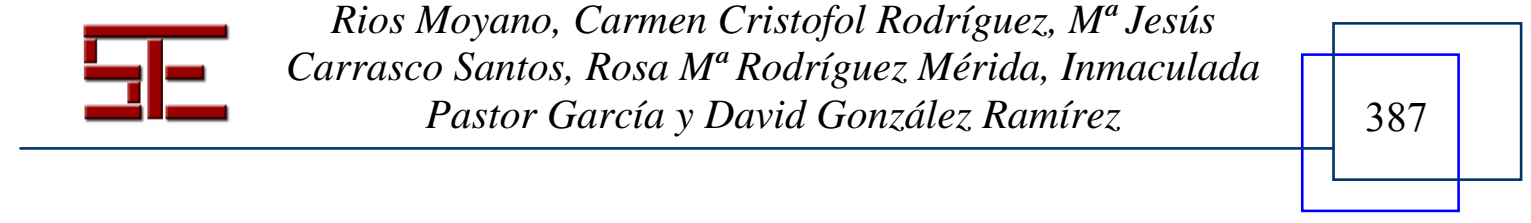




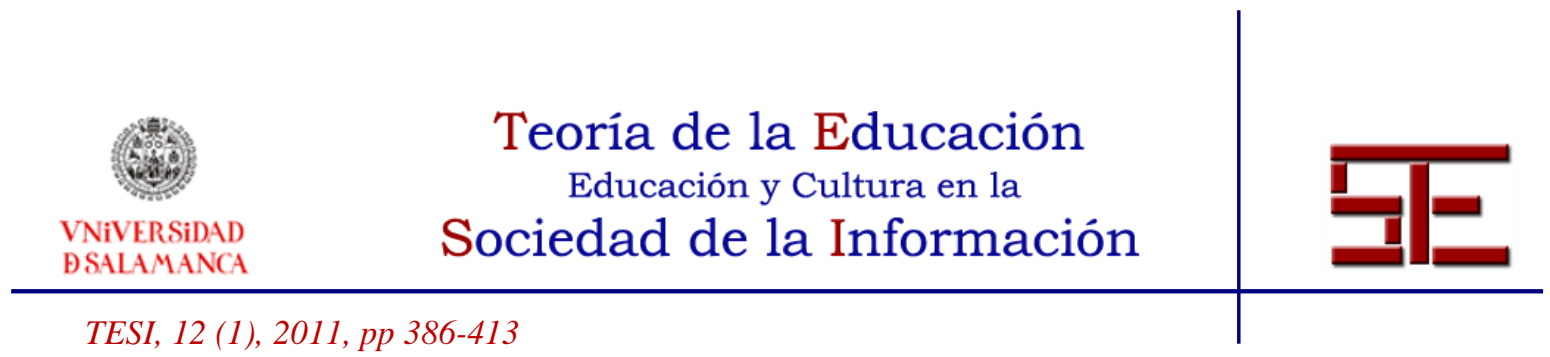

\section{LA INTERDISCIPLINARIEDAD EN LA EDUCACIÓN SUPERIOR: PROPUESTA DE UNA GUÍA PARA EL DISEÑO DE JUEGOS DE ROL}

Fecha de recepción: 14/09/2010; fecha de aceptación: 15/12/2010; fecha de publicación: 31/03/2011

Deborah García Magna
dgmagna@uma.es
Universidad de Málaga

Cristina Castillo Rodríguez

ccastillor@uma.es

Universidad de Málaga

Sonia Rios Moyano

srios@uma.es

Universidad de Málaga

Carmen Cristofol Rodríguez

carcrir@uma.es

Universidad de Málaga
$\mathrm{M}^{\mathrm{a}}$ Jesús Carrasco Santos mjcarrasco@uma.es Universidad de Málaga

Rosa Ma Rodríguez Mérida rmrodriguez@uma.es Universidad de Málaga

Inmaculada Pastor García ipastor@uma.es Universidad de Málaga

David González Ramírez davidgr@uma.es Universidad de Málaga

\section{1.- HACIA LA UNIVERSIDAD DEL SIGLO XXI}

En general, las universidades europeas han seguido durante décadas el modelo continental o napoleónico, o, como se suele decir, un modelo basado en estudiar mucho durante pocos años para trabajar toda la vida. Este modelo se refiere a aquella universidad que ha priorizado los contenidos, justificando que los conocimientos constituyen el aspecto más importante del proceso educativo y presuponiendo que estos conocimientos, adquiridos a través de las distintas asignaturas, permiten alcanzar un nivel de conocimiento, competencias, habilidades, aptitudes y actitudes necesarias para cada titulación. El proceso de enseñanza aprendizaje ha recaído durante décadas en el profesor, puesto que él es quien debía transmitir su conocimiento en un tiempo determinado y limitado por la unidad de medida adoptada ${ }^{1}$.

Esta necesidad de reforma universitaria no es nueva, aunque bien es cierto que los acuerdos europeos han iniciado definitivamente un cambio irreversible en la educación superior. Estas mismas reflexiones sobre la necesidad de cambio en la Universidad ya las abordaba casi a mediados del siglo pasado Ortega y Gasset $(1930,39)$ en su trabajo sobre la Misión de la Universidad, en el cual afirmaba que ta raíz de la reforma

Deborah García Magna, Cristina Castillo Rodríguez, Sonia Rios

$388 \pi \begin{gathered}\text { Moyano, Carmen Cristofol Rodríguez, Ma Jesús Carrasco } \\ \begin{array}{c}\text { Santos, Rosa Ma Rodríguez Mérida, Inmaculada Pastor García y } \\ \text { David González Ramírez }\end{array}\end{gathered}$




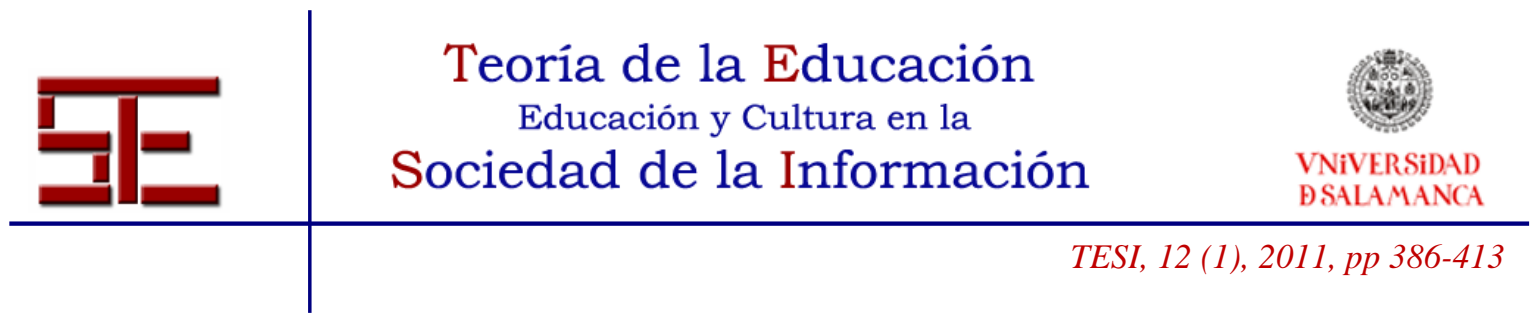

universitaria está en acertar plenamente con su misión”. Parece una ingenuidad que casi un siglo después sigamos planteándonos debates muy similares. Precisamente, en el Informe Universidad 2000 (Bricall, 2000²) se dedica un apartado íntegro a estas cuestiones, a saber, Cambios en la misión de la universidad, que hemos de entender en el sentido de adaptar la Universidad a los nuevos tiempos; y eso es lo que el docente universitario se plantea hacer utilizando las herramientas y recursos propios de su quehacer profesional. La Universidad ha de formar al ciudadano para el consumo cultural y científico, así como reforzar el pensamiento del hombre ante tanto exceso de información. Por su parte, Morin (2001, 22 23) plantea que

\footnotetext{
La reforma del pensamiento permitiría el pleno empleo de la inteligencia para responder a estos desafíos y facilitaría la unión de las dos culturas separadas. Se trata de una reforma, no programática, sino paradigmática, que concierne a nuestra aptitud para organizar el conocimiento. [...] La reforma de la enseñanza debe conducir a la reforma del pensamiento y la reforma del pensamiento a la reforma de la enseñanza.
}

La Universidad del siglo XXI debe ser una Universidad flexible y abierta, modernizada, innovadora y competitiva en aras de responder a los retos que la sociedad, así como el desarrollo cultural, científico, técnico y profesional le demandan en cada momento histórico. Al mismo tiempo, es necesaria la independencia y la autonomía para mantener su capacidad de crítica y salvaguardar el equilibrio entre sus componentes local y universal para enriquecer al estudiante y ciudadano.

El momento histórico en el que vivimos nos brinda una oportunidad única de redefinir nuestras metodologías docentes, de invertir el proceso de enseñanza aprendizaje dejando a un lado los antiguos métodos de transmisión del conocimiento basados casi exclusivamente en el aula a través del método expositivo, para incentivar el uso de otras metodologías más activas y participativas. Los docentes nos hemos preparado para un tipo de alumnado que se enmarca en el modelo establecido en la Declaración de Bolonia, es decir, un alumnado responsable, autónomo y colaborativo, por lo que se establecen un contexto y unos sujetos idóneos para llevar a cabo experiencias docentes que experimenten con posibles variaciones de los juegos de rol para atender de forma más específica a las necesidades y particularidades de nuestros títulos de grado.

\section{2.- METODOLOGÍAS ACTIVAS EN LA EDUCACIÓN SUPERIOR: LOS JUEGOS DE ROL}

Considerando la corriente pedagógica imperante —el constructivismo-, podemos entender el rumbo de la reforma metodológica propuesta en el Espacio Europeo de

Deborah García Magna, Cristina Castillo Rodríguez, Sonia

Rios Moyano, Carmen Cristofol Rodríguez, Mesús

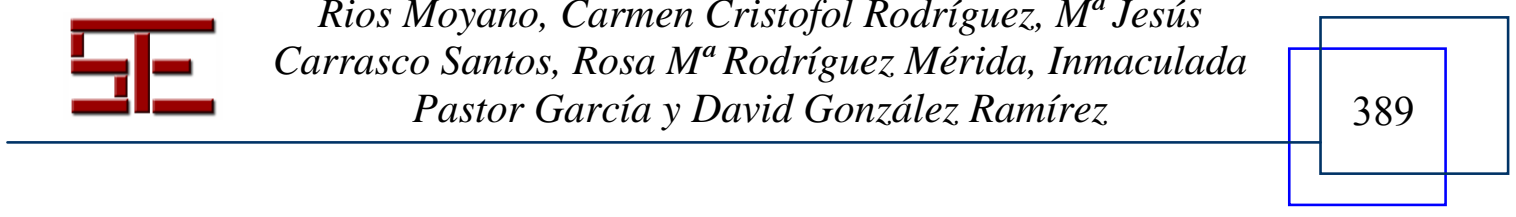




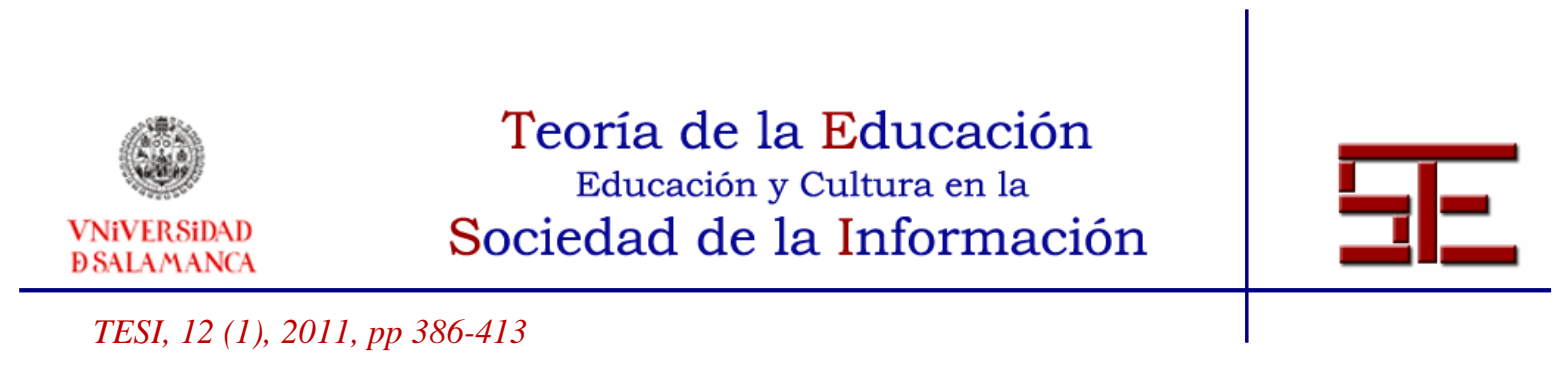

Educación Superior (en adelante, EEES). La palabra método proviene de los términos griegos meta (= objetivo) y odos (= camino, o forma de hacer algo). Por tanto, significaría la forma más adecuada de alcanzar una meta u objetivo. Por extensión, la metodología sería la ciencia o tratado del método, así como el sistema de métodos propio de cada ciencia particular. El método está estrechamente relacionado con los objetivos y contenidos, en tanto que es la forma de hacer o llevar a la práctica estos conceptos. Quizás, entre otras cuestiones, ésta sea una de las causas que ha propiciado un cambio en los modelos o plantillas de guías docentes necesarios para llevar a la práctica la reforma educativa. ¿Por qué? Pues porque tienen que quedar bien definidos todos y cada uno de los agentes implicados en el proceso de enseñanza aprendizaje.

Si consideramos esta premisa, nos daremos cuenta de la importancia de la utilización de las metodologías más adecuadas para cada título o disciplina, ya que se debe expresar necesariamente un proceso ordenado, regular, definido y lógico que permita precisamente avanzar por dicho camino para poder integrarse en una unidad significativa superior. Es cierto que son muchos los métodos existentes, y todos ellos son complementarios; es más, dependiendo del tipo de alumnado y del número de componentes del grupo, será más apropiado el uso de unas metodologías u otras. Precisamente, en el informe sobre educación presentado a la UNESCO y coordinado por Jacques Delors $(1996,34)$ se indicaba que los cuatro pilares en los que se basa la educación son los siguientes:

- Aprender a conocer, actividad más tradicional de la enseñanza a través de la transmisión de conocimientos del profesor al alumno, aunque complementada con nuevos aspectos.

- Aprender a hacer, visión práctica de la misma, mediante la capacitación del estudiante para enfrentarse a determinadas tareas.

- Aprender a vivir juntos, desarrollando la comprensión del otro y los valores del pluralismo y la percepción de las formas de interdependencia, sin renunciar a las propias ideas.

- Aprender a ser, supone el desarrollo de la personalidad, de la autonomía personal, del juicio y de la responsabilidad.

Si tenemos en cuenta que el concepto de aprender implica tanto la asimilación y reconstrucción de conocimientos como la adquisición y el uso de destrezas y el desarrollo de actitudes, estaremos apostando por la importancia del proceso de aprendizaje, o lo que es lo mismo, enseñar a nuestros alumnos a aprender a aprender.

Deborah García Magna, Cristina Castillo Rodríguez, Sonia Rios

$390 \quad \begin{gathered}\text { Moyano, Carmen Cristofol Rodríguez, Ma Jesús Carrasco } \\ \text { Santos, Rosa Má } M^{a} \text { Rodríguez Mérida, Inmaculada Pastor García y } \\ \text { David González, Ramírez }\end{gathered}$




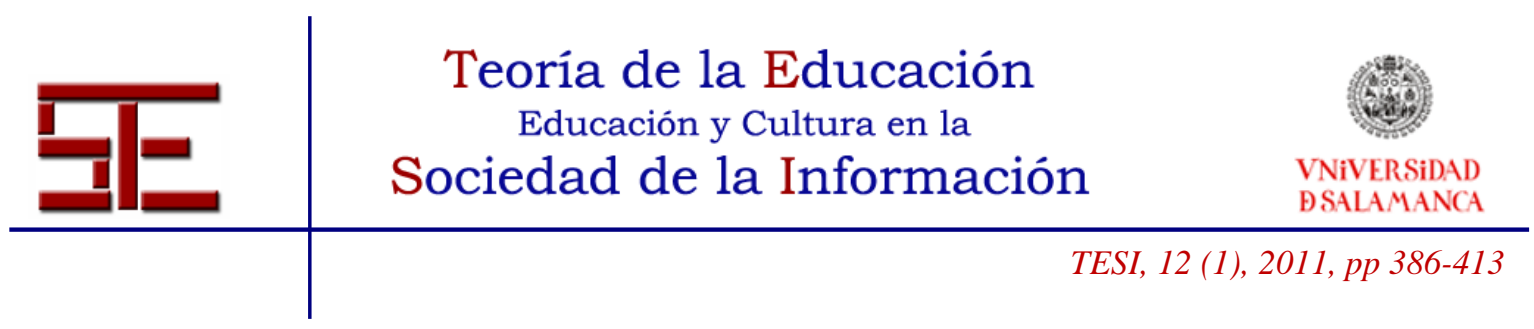

Aceptar esta frase como concepto e intentar llevarla a la práctica con las garantías de calidad exigibles en la educación superior, supone vertebrar todo nuestro trabajo en función de cómo podemos, como docentes, ayudar a nuestros alumnos para que aprendan de manera autónoma, es decir, enseñarles a aprender. En otras palabras, el objetivo no es sólo que aprendan los contenidos de nuestra materia, sino que adquieran una serie de competencias que puedan serles de utilidad durante toda su vida, lo que supone en la práctica que la persona que pase por nuestras aulas universitarias sepa seguir formándose durante toda su vida profesional teniendo como modelo las competencias que le enseñaron en la universidad y las estrategias necesarias para saber cómo adquirir un nuevo aprendizaje a lo largo de su vida (AAVV, 2005; Gil et al., 2004).

Tomando como base estas apreciaciones sobre la educación, el aprendizaje y el uso de metodologías adecuadas para conseguir unos fines y objetivos concretos, insistimos en la necesidad de utilizar metodologías activas y participativas que se adecuen a los objetivos generales de los títulos de grado y a los específicos de cada asignatura.

Asimismo, el uso de distintas metodologías activas en enseñanza superior permite al docente la incorporación de nuevos caminos en los que los estudiantes pueden adquirir los contenidos y competencias ligadas a una determinada materia en una disciplina dada, ya que de esta forma se está en consonancia con una de las propuestas del EEES para adaptar la educación a las demandas profesionales del mercado actual. Nos referimos a la consabida innovación, presente en todas las titulaciones de grado. Entre estas metodologías activas, que, por otro lado, favorecen el trabajo colaborativo, Pérez i Garcias (2002, 17 19) enumeran las siguientes: el trabajo en parejas, lluvia de ideas, rueda de ideas, debates, grupos de discusión, etc. Además de estas técnicas, se encuentra el uso de la simulación en el proceso de enseñanza aprendizaje, que, según Ruben (1999), basándose en los enfoques de aprendizaje de finales de los años 60, es una metodología que supera las limitaciones de la enseñanza tradicional. De hecho, y de acuerdo con este autor, el uso de las simulaciones como metodología educativa conlleva una mejora en el proceso de aprendizaje del propio estudiante, facilita la interactividad entre los estudiantes, el trabajo colaborativo y, en definitiva, un aprendizaje más activo.

Los enfoques de aprendizaje basados en la simulación tienen como objetivo primordial la representación de determinados aspectos de la vida real ${ }^{3}$. Un tipo de simulación es el llamado juego de rol, como bien explican Lean et al. (2006, 228): previous studies

Deborah García Magna, Cristina Castillo Rodríguez, Sonia

Rios Moyano, Carmen Cristofol Rodríguez, M Jesús

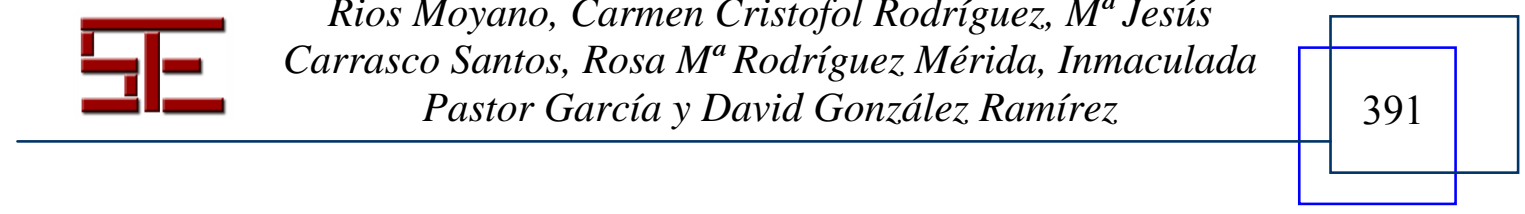




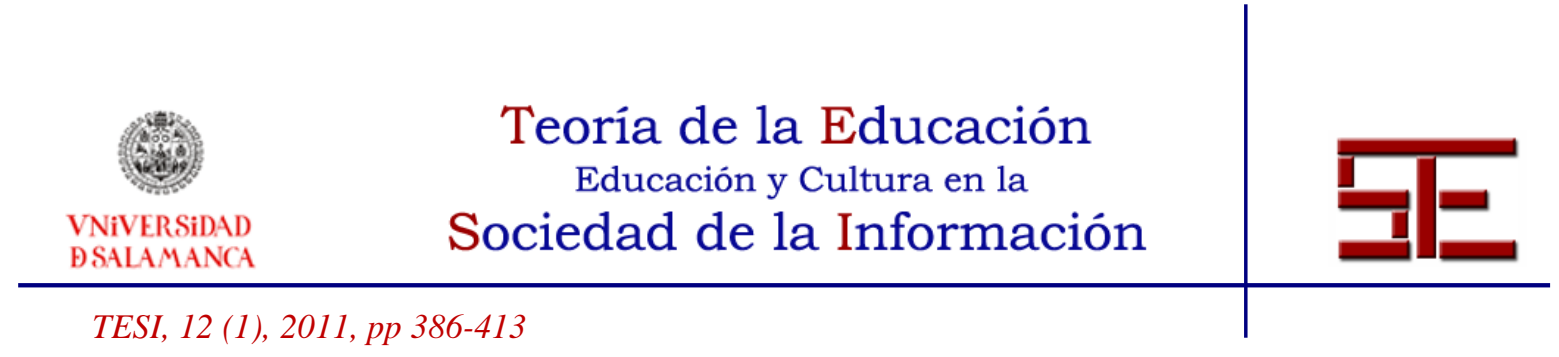

have identified three specific types of simulation based learning: role play, gaming and computer simulation" (Feinstein et al., 2002; Hsu, 1989).

Así, el juego de rol (role play o role playing) es la técnica en la que cada participante desempeña un papel concreto en el contexto de una situación simulada siguiendo determinadas reglas y en la que interactúa con otros participantes del mismo juego de simulación.

Lean et al. (2006) afirman que los juegos de rol se emplean en numerosas disciplinas tales como geografía (Oberle, 2004), lenguas extranjeras (Ladousse, 1987), trabajo social (Moss, 2000), ciencias (Sleigh, 2004), entre otras, siendo una de las técnicas de simulación de uso cada vez más extendido en la Universidad (León Vegas, 2009). No obstante, y aunque esta metodología se presenta como una herramienta útil a la par que rentable para que docentes y discentes se beneficien del proceso de enseñanza aprendizaje, lo cierto es que, según Lean et al. (2006, 228), existe muy poca literatura empírica sobre el uso de los juegos de rol en enseñanza superior (Armstrong, 2003).

Como bien afirman Jiménez y Moncholi $(2009,4)$ ta sociedad ya ha tomado conciencia de que la creatividad es un derecho y una exigencia social"; esta creatividad, indispensable en muchas disciplinas, puede desarrollarse a través de la técnica de juegos de rol, con la que, además, se potencia el aprendizaje y se suscita la curiosidad de los jugadores. Los juegos de rol, a pesar de que los personajes y la situación que se simula vienen definidos a priori, atendiendo a determinadas reglas impuestas por un director (normalmente el profesor o grupo de profesores que imparten una determinada materia), no exigen un diálogo concreto a los jugadores, siendo éstos libres para poder reconducir la situación o problemática planteada en aras de encontrar ciertas soluciones.

Sin embargo, es bastante sorprendente que no existan estudios empíricos a partir de la aplicación de esta técnica de innovación docente, tal y como ya hemos mencionado. Una de las razones por las cuales pensamos que no se le ha conferido la importancia que merece, más aún si consideramos las ventajas de su aplicación en el aula universitaria, es que no existen pautas generales sobre cómo abordar el juego de rol, así como las variables que se han de tener en cuenta a la hora de diseñarlo. Conscientes de estas dificultades, hemos decidido dirigir la investigación que ha dado pie al presente artículo, a elaborar una guía que facilite al docente la aplicación de esta metodología. Partimos, además, de que la gran fortaleza de la técnica de juegos de rol con respecto a otras metodologías activas es su capacidad para simular situaciones profesionales de carácter pluridisciplinar, aspecto que no se aborda de manera satisfactoria en el ámbito

Deborah García Magna, Cristina Castillo Rodríguez, Sonia Rios

$392 \quad \begin{gathered}\text { Moyano, Carmen Cristofol Rodríguez, Má Jesús Carrasco } \\ \text { Santos, Rosa Ma Rodríguez Mérida, Inmaculada Pastor García y } \\ \text { David González Ramírez }\end{gathered}$




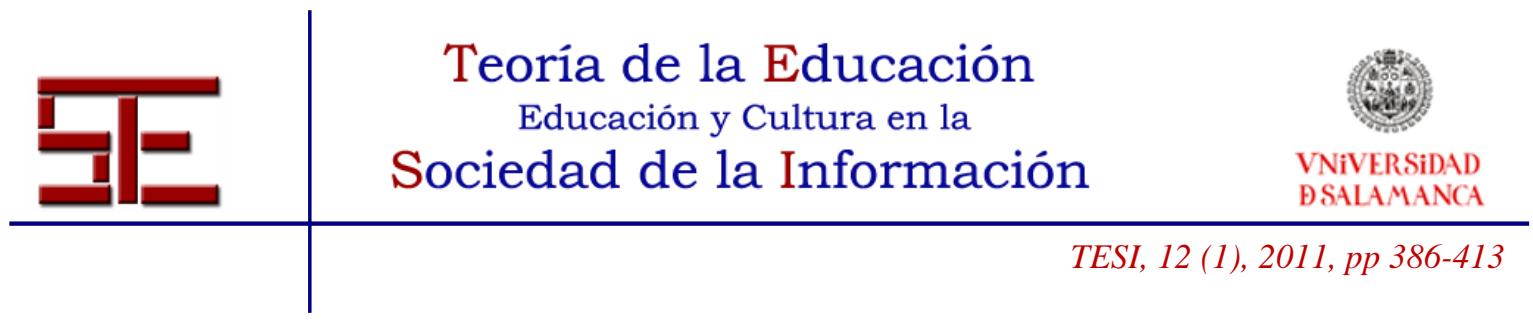

universitario y que consideramos fundamental en la formación de unos estudiantes que en el futuro tendrán que relacionarse con sujetos de diferente formación y perfil profesional.

\section{3.- LA INTERDISCIPLINARIEDAD EN EL SISTEMA EDUCATIVO}

La realidad laboral con la que se encuentran los estudiantes cuando terminan su etapa universitaria está llena de matices. Los problemas cotidianos necesariamente deben de abordarse desde perspectivas multidimensionales y a los profesionales del presente ya se les exige que adquieran una formación complementaria a la disciplina de la que son expertos o que, al menos, trabajen en colaboración con otros compañeros de distinto perfil. Por ello, es responsabilidad de la Universidad afrontar el reto de preparar a los profesionales del futuro para que adquieran competencias transversales que les permitan salir de sus conocimientos compartimentados, capacitándoles para afrontar con una visión más amplia la realidad en la que han de desarrollar su actividad.

\section{1.- Definición de interdisciplinariedad}

Según la RAE, el concepto interdisciplinario se define como -dicho de un estudio o de otra actividad: Que se realiza con la cooperación de varias disciplinas". Por su parte, Senge (1996) considera que una disciplina es un cuerpo teórico y técnico que debe ser estudiado y dominado para llevarse a la práctica y que permite adquirir aptitudes y competencias, lo que supone un compromiso constante de aprendizaje.

La interdisciplinariedad desde el punto de vista pedagógico fue una de las ideas principales en el seno de la Teoría Crítica ${ }^{4}$. Además, de acuerdo con Hernández Díaz $(2000,115)$, la pedagogía crítica se caracterizaría por un rechazo a considerar que el conocimiento sólo tenga un valor instrumental, reconociendo la necesidad de que los docentes empleen sus dotes interpretativas y consigan localizar y evitar las interpretaciones ideológicamente distorsionadas, ofreciéndoles explicaciones teóricas que les ayuden a identificar y superar las situaciones injustas, mediante el estudio previo del marco social, partiendo, en definitiva, de la idea de que es posible ayudar a los docentes a cambiar su forma de enseñar, es decir, en palabras de la autora: \#na investigación educativa que no sea sobre la educación sino para la educación".

Por su parte, Posada Álvarez (2004) hace especial hincapié en la importancia de la integración disciplinar como base de una flexibilización curricular para formar profesionales universales que sean capaces de enfrentarse a las nuevas transformaciones de los mercados (concretadas en competencias y conocimientos), debido a que la

Deborah García Magna, Cristina Castillo Rodríguez, Sonia

Rios Moyano, Carmen Cristofol Rodríguez, Ma Jesús

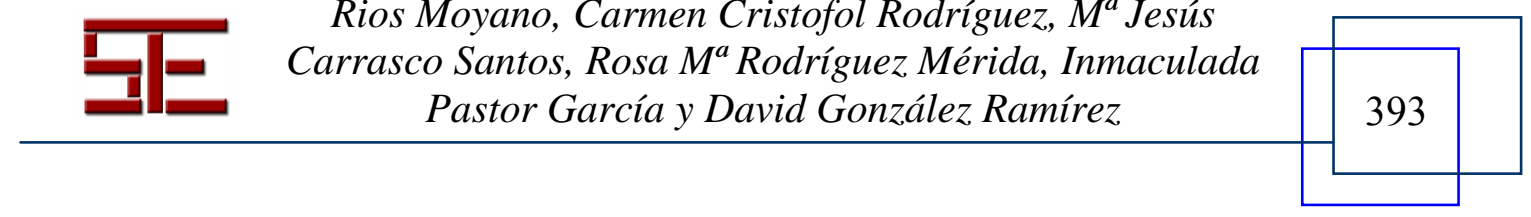




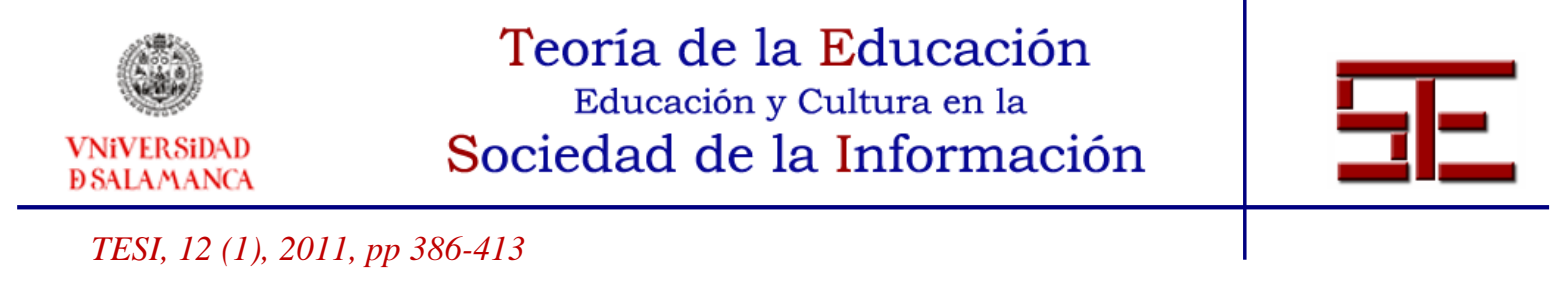

formación basada en competencias conlleva la integración de disciplinas, conocimientos, habilidades, prácticas y valores.

Piaget (1979) propone las siguientes dimensiones de integración disciplinar:

- Multidisciplinariedad: es el nivel inferior de integración, esto es, se trataría de la primera fase de formación de equipos de trabajo interdisciplinario, por lo que estos grupos de diferentes ámbitos trabajarían paralelamente, sin que la interacción producida cambie o enriquezca las disciplinas implicadas.

- Interdisciplinariedad: es el segundo nivel de integración disciplinar, en el que hay una verdadera reciprocidad en el intercambio de conocimiento. Este nivel, por tanto, implica la transformación de los conceptos y terminologías fundamentales, así como de metodologías de investigación y de enseñanza. Se trata de una integración recíproca entre varias disciplinas, las cuales son a su vez modificadas dependiendo así unas de las otras, cuya cooperación aporta un enriquecimiento mutuo.

- Transdisciplinariedad: es el nivel superior de integración disciplinar, esto es, se trata de unificar epistemológica y culturalmente los diferentes ámbitos de estudio para tratar de conseguir sistemas teóricos sin fronteras sólidas, fundamentadas en objetivos comunes.

En este sentido de interacción disciplinar, define el concepto de interdisciplinariedad Japiassu (1976), para quien consiste en un trabajo que se lleva a cabo en común, a partir de la cooperación de varias disciplinas, cuyos conceptos, metodologías, procedimientos, datos y la propia organización, inherente a cada una de ellas, deben también interaccionar.

Además, Morin (1994) considera que la interdisciplinariedad sería un proceso complejo en el que prima la interacción entre eventos, acciones y problemas del mundo actual. En definitiva, se trata de la profunda imbricación entre varias ciencias para dar lugar a un paradigma menos rígido que dé cuenta de esa complejidad inherente en la nueva sociedad donde el conocimiento no esté encasillado en compartimentos estancos y aislados.

Es por ello que en nuestra propuesta de guía de elaboración y diseño de juegos de rol hemos usado el calificativo de interdisciplinario, ya que, en primer lugar, se trata de

Deborah García Magna, Cristina Castillo Rodríguez, Sonia Rios

$394 \quad \begin{gathered}\text { Moyano, Carmen Cristofol Rodríguez, Ma Jesús Carrasco } \\ \text { Santos, Rosa Má Rodríguez Mérida, Inmaculada Pastor García y } \\ \text { David González, Ramírez }\end{gathered}$




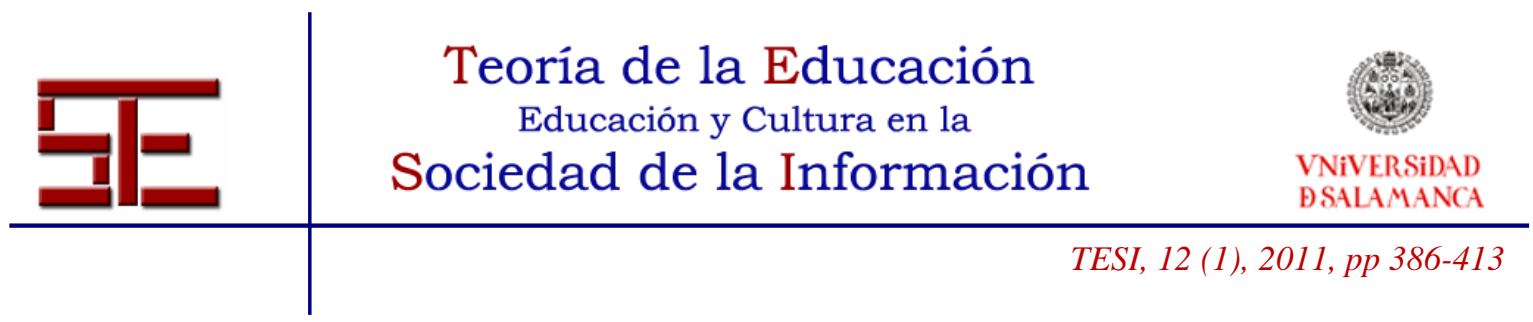

diseñar un juego de rol de integración disciplinar", puesto que pretendemos transformar una metodología de trabajo, los juegos de rol, con el fin de conseguir que los futuros profesionales adquieran un aprendizaje interdisciplinar, es decir, que aprendan a abordar las situaciones del mercado laboral de la forma más eficiente posible y desde varios puntos de vista. $Y$, en segundo lugar, porque se trata de conseguir que los alumnos de diferentes disciplinas, grados o áreas de conocimiento trabajen de manera conjunta para resolver un problema planteado.

\section{2.- ¿ ¿Por qué esta interdisciplinariedad?}

A partir del curso académico 20092010 ha comenzado a aplicarse, en la mayoría de las titulaciones de nuestro país, el proceso de Bolonia. Este proceso constituye una reforma en los sistemas de educación superior de 29 países de la Unión Europea con el objetivo general de construir el EEES. Para la implantación de dicho espacio se han establecido los principales objetivos para homologar la enseñanza superior europea a fin de fomentar la libre circulación de estudiantes, aumentar el atractivo internacional de la educación europea y permitir la acreditación de estudiantes y trabajadores por todo el territorio europeo. Así, con respecto a la enseñanza, existen tres niveles:

Nivel 1: Título de grado, que capacita para el acceso al mercado laboral

Nivel 2: Obtención de título máster

Nivel 3: Obtención de título doctorado

Además de estos tres niveles, se implanta un Suplemento Europeo al título donde se describen con precisión las capacidades adquiridas por el alumno durante sus estudios. Según la Declaración de Bolonia, el diseño de los planes de estudio debe ofrecer una formación adecuada para el desarrollo de los perfiles profesionales que previamente han sido definidos para la titulación. Dicha formación debe permitir a los estudiantes adquirir una serie de conocimientos y habilidades que, de acuerdo con la terminología empleada en dicha Declaración de Bolonia, se denominan competencias, las cuales pueden clasificarse en tres tipos, a saber, específicas, genéricas y transferibles.

Las competencias específicas están relacionadas con el área o áreas de conocimiento y práctica profesional de la titulación, confiriendo cierta identidad y consistencia al programa de aprendizaje. Dentro de este grupo de competencias específicas también se distinguen otras, que podríamos definir como subcompetencias específicas; nos referimos a las básicas y a las especializadas. Además, todas las competencias específicas requieren la inclusión en el plan de estudios de una materia o asignatura.

Deborah García Magna, Cristina Castillo Rodríguez, Sonia

Rios Moyano, Carmen Cristofol Rodríguez, M Jesús

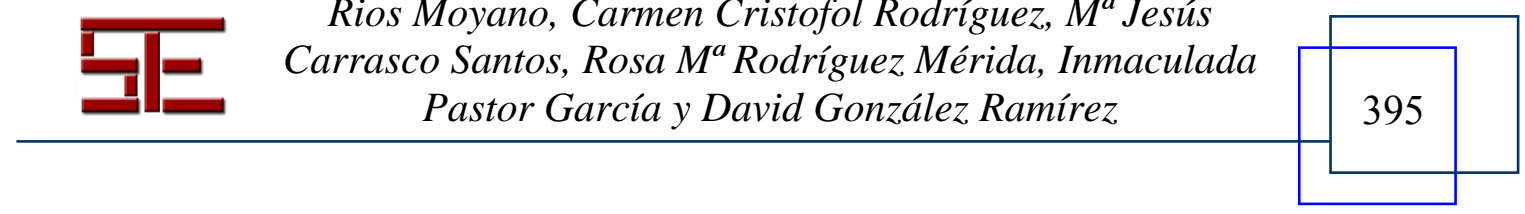




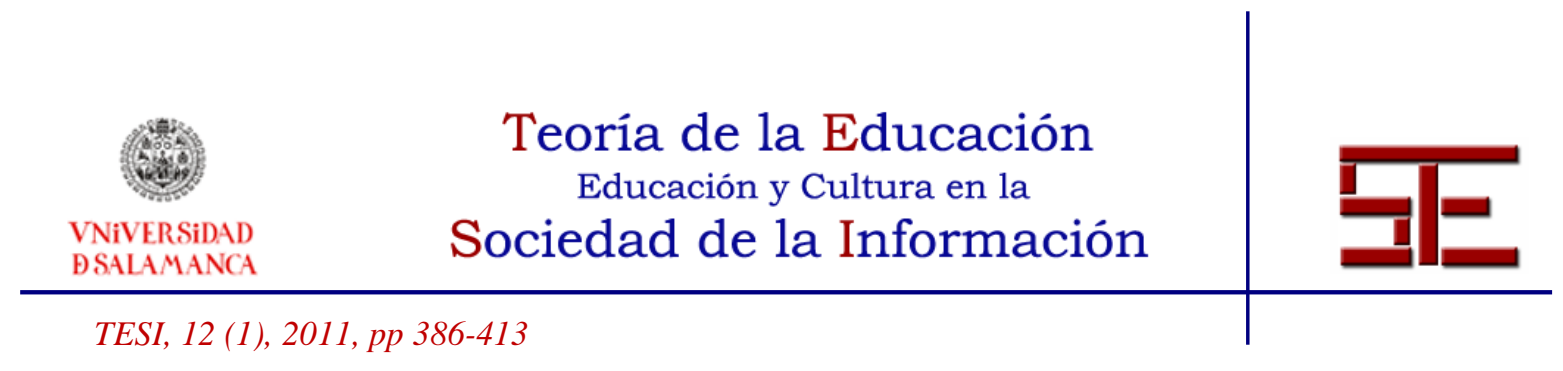

Las competencias genéricas o transversales son las que hacen referencia a los atributos que debería tener un grupo social particular. Estas competencias, a su vez, se subdividen en competencias técnicas o instrumentales, sistémicas y personales. Las primeras engloban aquellas competencias que tienen que ver con la capacidad de análisis y síntesis, resolución de problemas, comunicación oral y escrita en la lengua nativa, así como en otra lengua extranjera y la toma de decisiones, entre otras. Las competencias transversales sistémicas muestran las capacidades y habilidades que deben adquirir los egresados en lo que respecta al aprendizaje y trabajo autónomos, la creatividad e innovación, la adaptación a nuevas situaciones y aplicación práctica de los conocimientos teóricos. Y, por último, entre las competencias transversales personales destacan, a modo de ejemplo, el razonamiento crítico, el trabajo colaborativo, así como en contextos internacionales, la capacidad de comunicación con personas no expertas en la materia, entre otras.

En cuanto a las competencias transferibles, son aquéllas necesarias para estrechar la brecha existente entre la teoría y la realidad y, por consiguiente, el mercado laboral. Estas competencias se adquieren mediante la inclusión de metodologías que promuevan actividades prácticas dirigidas fundamentalmente a la resolución de problemas profesionales reales.

El esquema seguido para la determinación de la propuesta de los futuros planes de estudios, debe partir del consenso entre profesionales y académicos acerca de sus contenidos básicos, sin olvidar el carácter prioritario de empleabilidad exigido por la Declaración de Bolonia.

\section{4.- EL JUEGO DE ROL INTERDISCIPLINAR: UNA GUÍA PARA EL DOCENTE}

Resulta manifiesta, por todo lo expuesto, la necesidad en el ámbito educativo actual de favorecer contextos de aprendizaje interdisciplinares que promuevan la adquisición de las competencias necesarias para desempeñar la labor profesional de manera más eficiente. Por ello, proponemos, a continuación, una metodología que pretende precisamente colocar al alumno en un contexto que recree una situación profesional en la que deba necesariamente interactuar con otros expertos de perfil y formación distintos al suyo, tal como le sucederá en la vida real.

Deborah García Magna, Cristina Castillo Rodríguez, Sonia Rios

$396 \quad \begin{gathered}\text { Moyano, Carmen Cristofol Rodríguez, Ma Jesús Carrasco } \\ \text { Santos, Rosa M Modríguez Mérida, Inmaculada Pastor García y } \\ \text { David González, Ramírez }\end{gathered}$




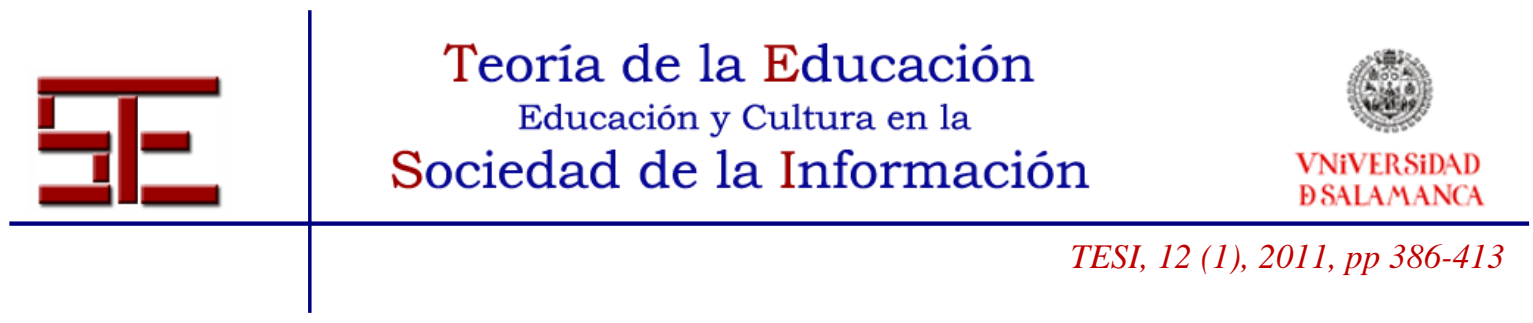

\section{1.- El juego de rol interdisciplinar como metodología innovadora}

Nuestra propuesta de uso del juego de rol interdisciplinar conlleva la adopción de una metodología innovadora que contiene todas las ventajas del método del caso, aunque con un enfoque mucho más dinámico, añadiendo el elemento innovador de la interdisciplinariedad, que entendemos es fundamental en la formación de profesionales preparados para el mercado laboral actual.

No nos pasa desapercibido que el diseño de cualquier juego de rol puede ser complejo, como ya venimos afirmando a lo largo de todo el artículo, ya que incluye no sólo el supuesto práctico a resolver, sino también el contexto y los perfiles de los personajes que los alumnos representarán, además de la preparación y el desarrollo de la actividad, que conllevará normalmente la implementación del juego en varias sesiones. Si a todo ello le unimos el componente interdisciplinar, que implicará la puesta en común con otros docentes de distintas áreas de conocimiento y el posterior desarrollo del juego, coordinando a los alumnos de las otras disciplinas, la tarea se vuelve aún más complicada y, sobre todo, requerirá, por parte de los docentes, una inversión en trabajo y tiempo que puede no resultar rentable. Por otro lado, si la actividad no está bien planificada, probablemente los alumnos también acaben empleando más tiempo del que se estime necesario y no consigan sacar el máximo provecho de la experiencia.

Por todo ello, hemos centrado nuestros esfuerzos en elaborar una guía que sirva de modelo al docente para diseñar de manera eficiente un juego de rol interdisciplinar, sacando el mayor rendimiento posible a su tiempo y trabajo.

\section{2.- $\quad$ Modelo para el diseño de juegos de rol interdisciplinares}

El modelo que proponemos repasa de manera exhaustiva todos los parámetros que entendemos fundamentales para planificar y desarrollar de manera eficaz esta metodología. Se abordan las diez variables que se deben tener en cuenta tanto en el diseño de la estrategia docente, como en su puesta en práctica y posterior evaluación. Todas las variables y subvariables se encuentran estandarizadas, de manera que son comunes a cualquier disciplina. No se debe olvidar que se trata de un esquema que pretende guiar al docente, siendo éste quien después deberá adaptar el juego resultante a su área de conocimiento, ideando un supuesto práctico concreto.

A continuación presentamos el esquema, así como la explicación de las variables que éste engloba:

Deborah García Magna, Cristina Castillo Rodríguez, Sonia

Rios Moyano, Carmen Cristofol Rodríguez, $M^{a}$ Jesús

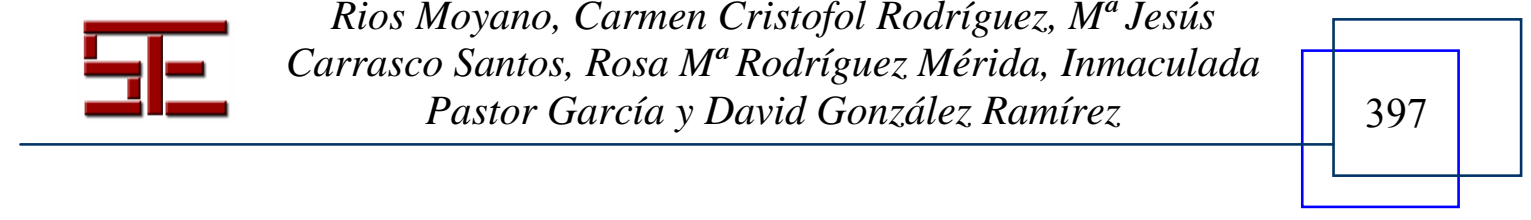




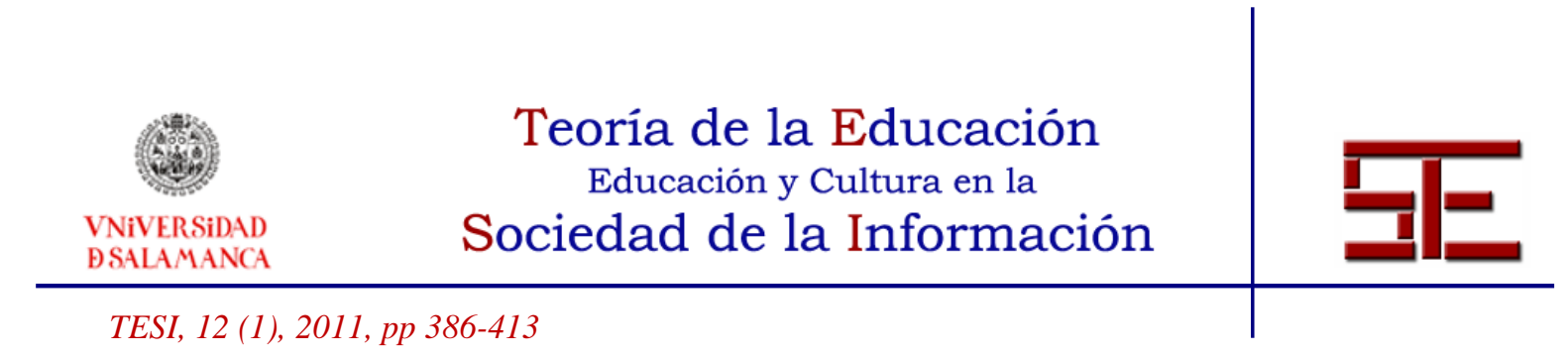

VARIABLE 1. TAMAÑO DEL GRUPO

- Pequeño (menos de 20 personas)

- Mediano (entre 20 y 50 personas)

- Grande (más de 50 personas)

VARIABLE 2. PERFIL DE LOS ESTUDIANTES PARTICIPANTES

- 2.1. Según disciplina:

- Pseudoheterogéneo

○ Heterogéneo

- 2.2. Según nacionalidad:

○ Nacional

- Internacional

- EEES

- Otro plan de estudios

- 2.3. Según nivel de estudios:
- Grado
- Posgrado
○ Titulaciones propias (Experto, Especialista Universitario, etc.)

\section{VARIABLE 3. ORGANIZACIÓN DE LA ACTIVIDAD}

- 3.1. Actividad integrada en la programación docente oficial de la asignatura

- Plazos y requisitos formales para su inclusión

- Reunión con los docentes de la misma área de conocimiento

- Acuerdo sobre contenidos e inclusión en la programación docente

- Aprobación por el órgano competente

- Si grupo heterogéneo:

- Reunión previa con docentes de las disciplinas participantes, para que sigan los mismos trámites en sus respectivas áreas

- 3.2. Actividad formativa autónoma

- Taller (informal)

- Convocatoria no oficial

- Plazos de inscripción para alumnos

- Proceso de selección de participantes

- Solicitud de espacio para desarrollar la actividad

- Previsión del horario

- Información sobre el programa de la actividad

- Seminario (formal)

Deborah García Magna, Cristina Castillo Rodríguez, Sonia Rios Moyano, Carmen Cristofol Rodríguez, $M^{a}$ Jesús Carrasco 


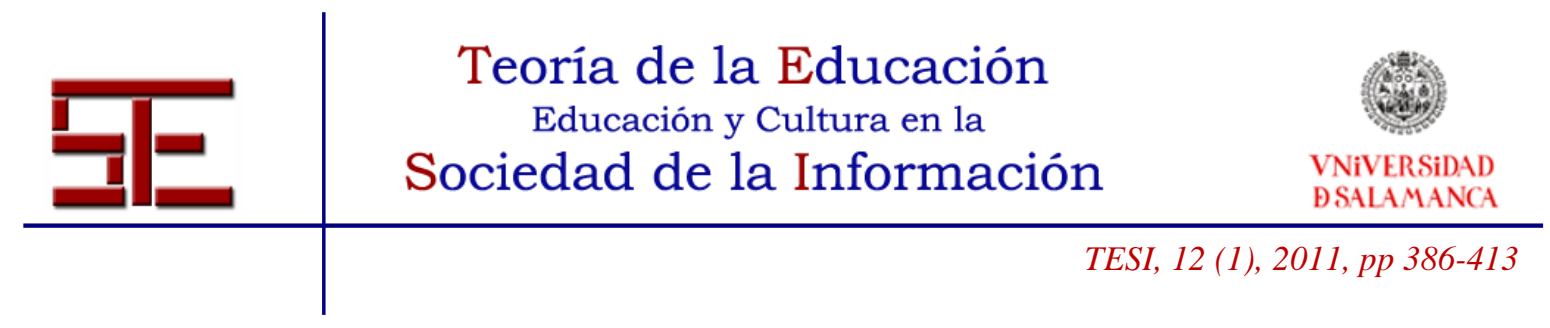

- Trámites administrativos para su concesión

- Convocatoria oficial

- Plazos de matrícula

- Proceso de selección de participantes

- Publicación del programa de la actividad

VARIABLE 4. CARACTERÍSTICAS DEL JUEGO

- 4.1. Participación

- Obligatoria

- Voluntaria

- 4.2. Roles

○ Jugadores

- No jugadores

- 4.3. Explicación del supuesto práctico y las reglas del juego

o Virtual

- Foros

- Colectivo

- Presencial

- Grupos

- Reparto de fichas

- Pizarra virtual

- Otros

- 4.4. Contexto

○ Virtual

- Diseño del espacio (imágenes)

- Explicación del contexto (texto)

○ Presencial

- Atrezzo

- Decoración del espacio

- Disfraces de los personajes

- Sin atrezzo

- Adecuación del aula (mesas, sillas, pizarra)

- 4.5. Temporalización

- Planificación de la actividad por el docente

- Número de horas para organizar la actividad (trámites administrativos, reuniones con otros docentes)

Deborah García Magna, Cristina Castillo Rodríguez, Sonia

Rios Moyano, Carmen Cristofol Rodríguez, M Jesús

Carrasco Santos, Rosa M $M^{a}$ Rodríguez Mérida, Inmaculada Pastor García y David González Ramírez 


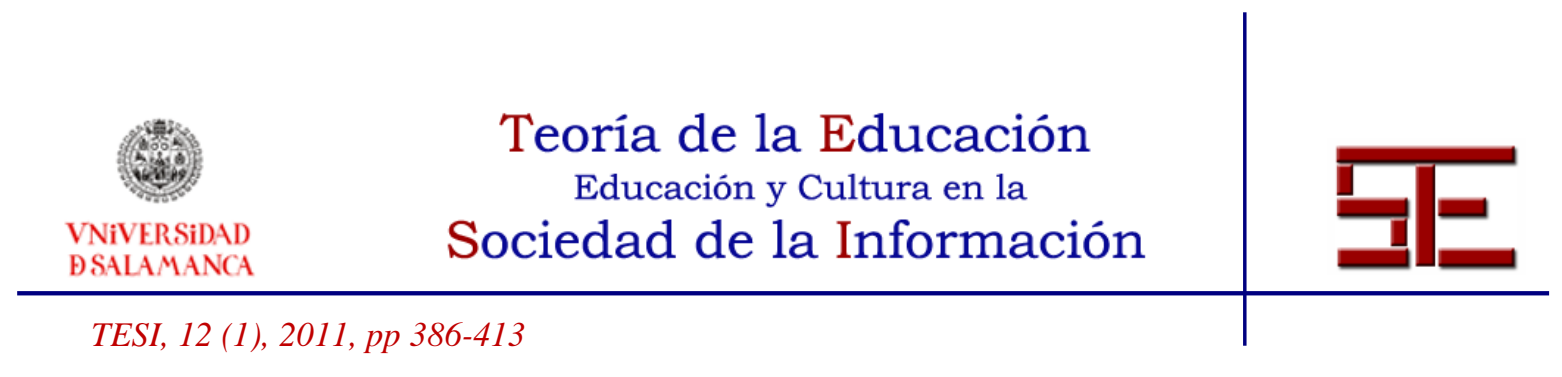

- Número de horas para diseñar el juego (supuesto práctico, personajes, contexto)

- Plazos para los estudiantes (convocatoria, solicitudes de participación, publicación de admitidos)

○ Presentación de la actividad a los estudiantes

- Si presencial

- Número de sesiones

- Si virtual

- Plazo

- Preparación del juego

- Si presencial

- Número de sesiones/horas (docente y estudiantes)

- Número de sesiones/horas (estudiantes solos)

- Si virtual

- Plazo

○ Ejecución del juego

- Si presencial

- Si virtual

- Número de sesiones

- Plazo

- Evaluación del proceso de aprendizaje

- Plazos

- Número de sesiones de debate entre docentes

- Valoración del desarrollo de la actividad

- Recopilación de la información

- Número de sesiones (si debate reflexivo en grupo)

- Plazo (si encuestas, portafolio, diario, etc.)

- Valoración de la información

- Plazo

- Número de sesiones de debate entre docentes

VARIABLE 5. TUTORIZACIÓN

- 5.1. Lugar

- Presencial

- En el aula

- En el despacho del docente

- Virtual

Deborah García Magna, Cristina Castillo Rodríguez, Sonia Rios

Moyano, Carmen Cristofol Rodríguez, $M^{a}$ Jesús Carrasco

Santos, Rosa M $M^{a}$ Rodríguez Mérida, Inmaculada Pastor García y

400 David González Ramírez. 


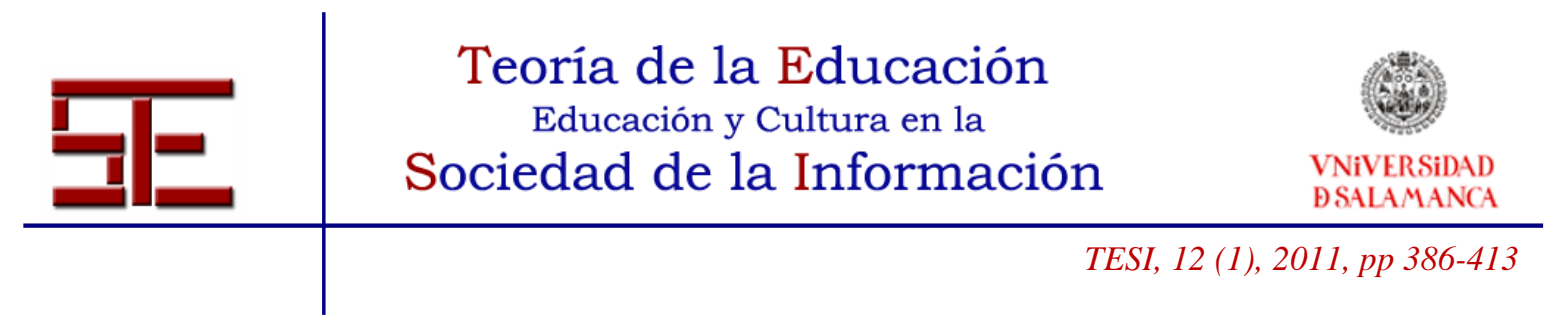

- En la plataforma moodle

- En el contexto de una red social

- 5.2. Forma

- Vía correo electrónico

- Individual

- Colectivo

VARIABLE 6. EVALUACIÓN DEL JUEGO

- 6.1. Quién evalúa

○ El docente

- Cada profesor evalúa los roles de su disciplina

- Todos los profesores emiten evaluación en conjunto sobre todos los roles (competencias transversales)

- Los estudiantes (mediante informe o cuestionario)

- Participantes

- Espectadores

- 6.2. A quién se evalúa

- Al estudiante

- Al profesor

- 6.3. Qué se evalúa

- Resolución del supuesto práctico

- Informes por escrito entregados por el alumno

- Sobre el rol a representar

- Sobre el supuesto práctico

- Representación

- En el acto

- Posteriormente (vídeo)

VARIABLE 7. ALUMNOS SIN ROL

- Evalúan la actividad

- Realizan un informe sobre lo que han visto

- Valoran la actuación de sus compañeros

- Se organizan en grupos que asesoran a cada personaje

- Se les evalúa sobre el contenido de la materia representada

- Son meros espectadores

- No están presentes cuando se desarrolla el juego

Deborah García Magna, Cristina Castillo Rodríguez, Sonia

Rios Moyano, Carmen Cristofol Rodríguez, $M^{a}$ Jesús

- Carrasco Santos, Rosa Ma Rodríguez Mérida, Inmaculada Pastor García y David González Ramírez 


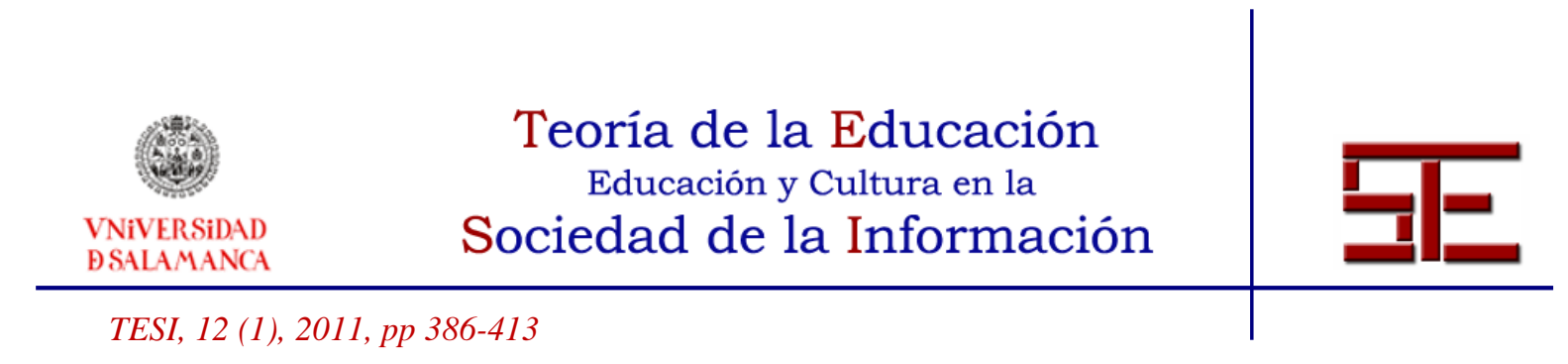

VARIABLE 8. MOTIVACIÓN PARA LOS ESTUDIANTES

- El juego de rol es una actividad metodológica más y el alumno descubre su valor de forma autónoma

- Se plantea como una forma de evaluación de toda o parte de la asignatura por la que participan:

○ Única

- Junto con otras formas de evaluación

- Premios

○ Libros

- Material relacionado con su disciplina que le pueda ser útil

o Otros

- Puntos extra en la asignatura por la que participen

- Créditos de libre configuración

- Certificado de aprovechamiento

VARIABLE 9. RETROALIMENTACIÓN PARA EL DOCENTE

- Encuestas de valoración a los alumnos participantes

- Revisión comparativa de calificaciones obtenidas antes y después de la puesta en práctica de esta metodología

- Comparativa de asistencia a clase antes y después de la puesta en práctica de la actividad

- Pruebas -exámenes, tests- sobre los contenidos tratados en el juego de rol

- Entrevistas personales a algunos de los alumnos o a todos ellos

- Diario que deberán llevar tanto los estudiantes como los profesores participantes

- Portafolio

- Sesiones de debate sobre el desarrollo de la actividad

VARIABLE 10. RECURSOS HUMANOS Y MATERIALES

- 10.1. Recursos materiales:

- Presencial:

- Sólo papel y bolígrafo

- Fichas:

- Personajes

- Contexto y situación real a resolver

- Atrezzo:

- Contexto (decoración)

Deborah García Magna, Cristina Castillo Rodríguez, Sonia Rios Moyano, Carmen Cristofol Rodríguez, $M^{a}$ Jesús Carrasco Santos, Rosa Ma Rodríguez Mérida, Inmaculada Pastor García y David González Ramírez. 


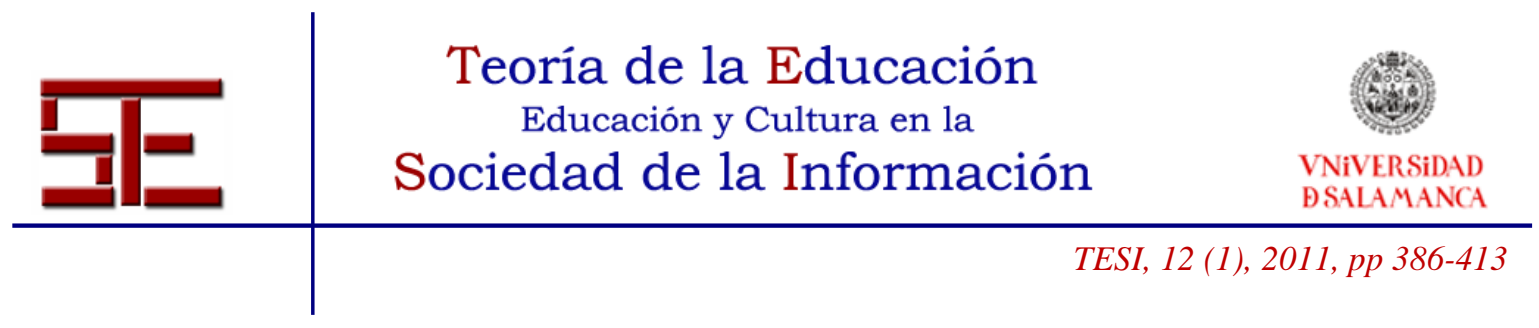

○ Virtual

- Indumentaria de los personajes

- Material bibliográfico de consulta

- Plataforma específica para la actividad y/o la propia de la asignatura

- Espacio en redes sociales

- 10.2. Recursos humanos:

- Profesorado y alumnos participantes

- Personal de apoyo (técnicos informáticos, personal a cargo de la biblioteca, otros profesores, otros alumnos, etc.), dependiendo de la dificultad y complejidad del juego.

Variable 1: Tamaño del grupo.

Esta actividad requiere una atención personalizada a cada alumno, no sólo en su preparación sino también en su ejecución y posterior evaluación, lo cual implica una alta inversión de tiempo para el docente. Por ello, es importante programarla en función del número de alumnos (teniendo en cuenta, además, la participación de estudiantes de otras disciplinas). En un grupo pequeño se puede plantear como una actividad de obligada participación para todos; sin embargo, en un grupo grande, resultará más eficaz diseñarla como actividad de carácter voluntario. Dependiendo del número de personajes que intervengan en el juego, se puede saber de antemano cuántos subgrupos distintos van a conformarse, de manera que se puedan prever otras variables, como por ejemplo, dónde y cuántas veces se representará el juego (espacio y tiempo necesarios).

Variable 2. Perfil de los participantes.

Es fundamental estudiar detenidamente quiénes son los alumnos que van a participar en la actividad, para diseñar un supuesto práctico óptimo en un doble sentido: por un lado, partiendo de las habilidades que ya tengan adquiridas $\mathrm{y}$, por otro, ayudándoles a desarrollar las competencias que necesiten en función de su futuro perfil profesional. Así, el grupo que va a realizar la actividad puede ser heterogéneo (cuando varios docentes organizan el juego de rol conjuntamente, aportando estudiantes de sus respectivas disciplinas) o pseudoheterogéneo (si hay un único docente que imparte una materia a un grupo de alumnos que proceden de diversas disciplinas, por ejemplo, en cursos de posgrado). Del mismo modo, es conveniente conocer el plan de estudios de la Universidad de origen de los estudiantes, es decir, su procedencia (nacional o internacional, en función de que las Universidades de origen sean o no del mismo país) y su nivel académico (si cursan estudios de grado, posgrado o de otro tipo).

Deborah García Magna, Cristina Castillo Rodríguez, Sonia

Rios Moyano, Carmen Cristofol Rodríguez, Mesús

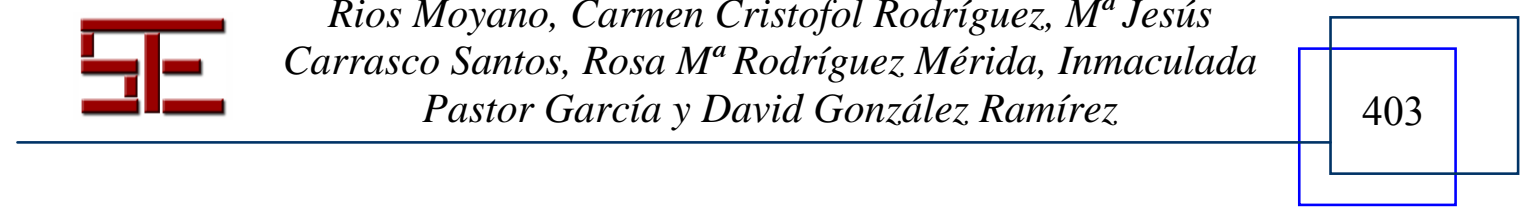




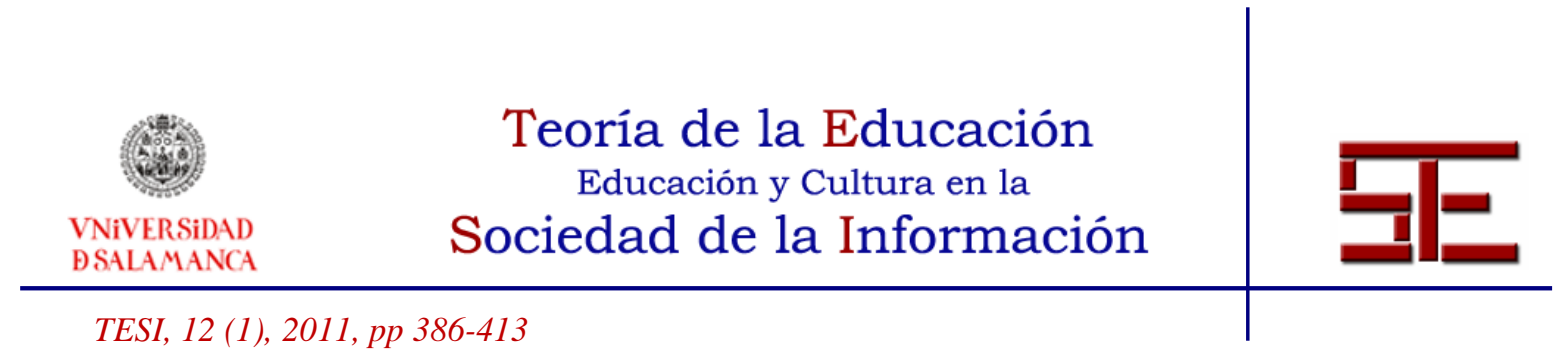

Variable 3. Organización de la actividad.

Se requiere un análisis previo de los trámites formales a seguir para que la actividad se integre correctamente con el resto de metodologías usadas por el docente. Si se pretende que la actividad entre en un programa oficial concreto, los profesores de las disciplinas participantes deberán incluirla en la programación docente de sus respectivas asignaturas y, por tanto, prever los plazos y trámites administrativos que establezca la Universidad, así como los requisitos de contenido de la metodología que han de plasmarse en el programa (número y distribución de créditos ECTS, forma de evaluación, etc.). Para ello, probablemente, tendrán que acordar unos contenidos mínimos con los demás docentes de sus respectivas áreas de conocimiento y se requerirá la aprobación formal del órgano de gobierno competente. Si se proyecta como una actividad formativa autónoma, al margen de la programación docente oficial, se puede organizar como un complemento extraacadémico (por ejemplo, un taller en el que puedan inscribirse alumnos de los docentes que organizan el juego) o como un curso formal independiente de la titulación (solicitando a la Universidad la organización de un seminario con dotación económica, aprovechando acuerdos Universidad-empresa, etc.). La complejidad de los trámites previos es distinta, aunque las variables a tener en cuenta serán parecidas: convocatoria (cartelería, servicios de información de la Universidad, publicación en prensa, etc.), plazos de inscripción o matrícula, proceso de selección de los participantes, publicación o información sobre el programa de la actividad y previsión del espacio donde se desarrollará y del horario que tendrá (sobre todo, en el caso del taller, ya que habrá que compatibilizar esta actividad con otras incluidas en la programación docente oficial de los alumnos participantes).

Variable 4. Características del juego.

Para obtener el máximo provecho de esta actividad, no sólo es preciso idear un buen supuesto práctico que sirva de hilo conductor del juego, sino que es imprescindible prever los detalles organizativos fundamentales. Por un lado, hay que decidir si la participación de los alumnos en la actividad va a ser obligatoria o no. Ello determinará otras variables como el sistema de evaluación, el espacio necesario, la distribución del tiempo, la previsión de actividades para los que no participen, etc. En el caso de una actividad formativa expresamente diseñada para poner en práctica esta metodología, es evidente que la participación de todos los estudiantes que se inscriben será obligatoria; sin embargo, si el juego de rol se integra en un programa junto con otras metodologías $\mathrm{y}$, sobre todo, si el grupo en el que se pretende aplicar es grande (o incluso mediano), será más eficiente plantearla como voluntaria. En cualquier caso, dentro del grupo de los que participan (ya sean todos los alumnos que tiene el docente o sólo algunos), y

Deborah García Magna, Cristina Castillo Rodríguez, Sonia Rios

$404 \quad \begin{gathered}\text { Moyano, Carmen Cristofol Rodríguez, Ma Jesús Carrasco } \\ \text { Santos, Rosa } M^{a} \text { Rodríguez Mérida, Inmaculada Pastor García y } \\ \text { David González, Ramírez }\end{gathered}$




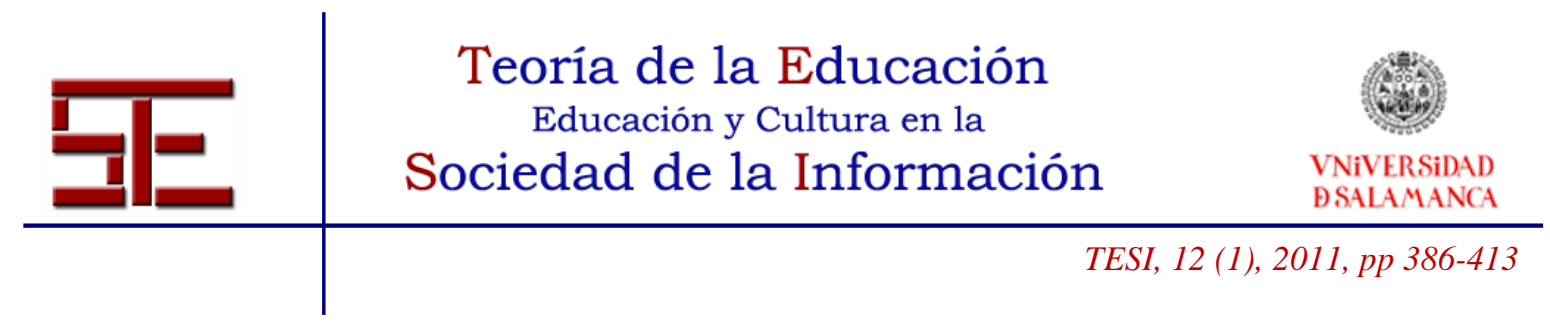

puesto que no podrán jugar todos simultáneamente (salvo que el grupo sea muy pequeño y el juego se represente sólo una vez), se formarán subgrupos de juego, de manera que normalmente los miembros de un subgrupo serán jugadores mientras se represente su juego, pero dejarán de serlo cuando le toque el turno a otro subgrupo: será preciso, por tanto, prever qué hacen los estudiantes que no juegan (variable 7). Por otra parte, es fundamental que todos entiendan muy bien en qué consiste la actividad. El supuesto práctico y las reglas del juego se pueden explicar de manera presencial (mediante fichas detalladas o usando la infraestructura con que cuente la sala) o virtual (utilizando foros para todo el grupo de participantes o para los diversos subgrupos). Hay que organizar con antelación de qué manera se va representar el juego (si el contexto será virtual o presencial, y si se va a utilizar algún tipo de atrezzo). Por último y quizás más importante a nivel organizativo, resulta esencial hacer una previsión lo más ajustada posible de la distribución del tiempo que va a durar la actividad en cada una de sus fases. Ello repercutirá de manera trascendental en la eficiencia de esta metodología.

Variable 5. Tutorización.

Será preciso desarrollar una acción tutorial coordinada desde el principio, no sólo para resolver las dudas que les surjan a los alumnos en la preparación y representación posterior de los roles que desempeñen en el juego, sino también para guiarles en la resolución del supuesto práctico que, en definitiva, deben superar en colaboración con los demás estudiantes. En este sentido, cada docente asesorará a los participantes sobre aspectos concretos de su disciplina, pero además, deberá tener en cuenta las competencias transversales que todos ellos deben adquirir y desarrollar, de manera que será precisa también una acción conjunta y coordinada con los demás docentes. Las tutorías, individuales o en grupo, se pueden llevar a cabo de manera presencial (ya sea en un aula o en el despacho del docente), o virtualmente (a través de la plataforma creada expresamente para la actividad, la de la asignatura de que se trate, una red social o mediante el correo electrónico).

Variable 6. Evaluación del juego.

Al desarrollarse el juego se pueden valorar una gran variedad de aspectos además de los conocimientos propios adquiridos pertenecientes a la asignatura: la responsabilidad que toma el alumno y formalidad en su actitud; si es capaz de trabajar en grupo; si es rápido en escoger entre las opciones posibles; la capacidad de improvisación y espontaneidad o si presenta una actitud sociable. Todas ellas son capacidades que hay que tener en los juegos de rol al igual que en el trabajo en equipo. Es de gran importancia la evaluación del valor que puede aportar a los alumnos el juego realizado de forma interdisciplinar y

Deborah García Magna, Cristina Castillo Rodríguez, Sonia

Rios Moyano, Carmen Cristofol Rodríguez, M Jesús

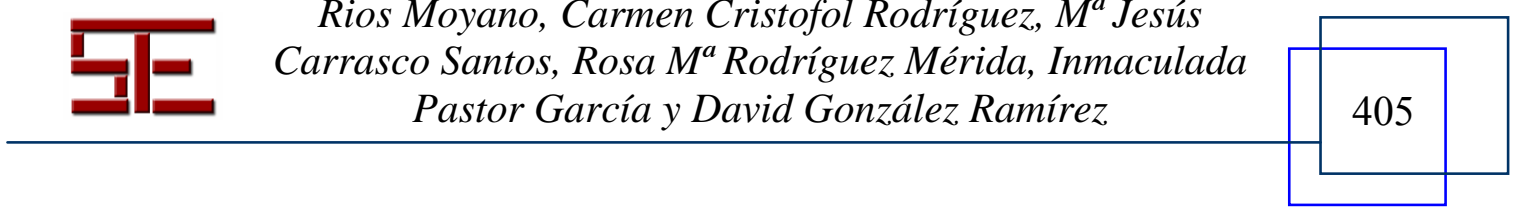




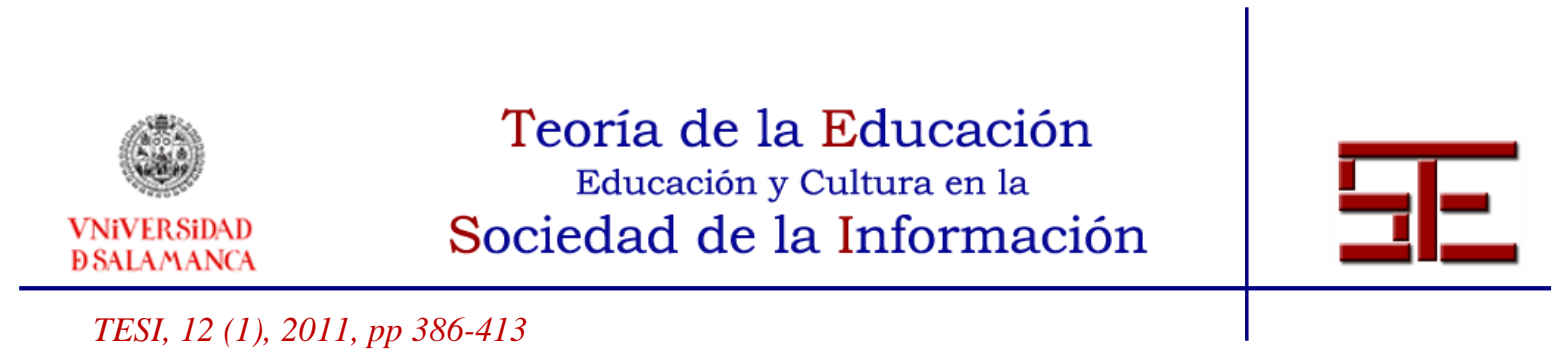

el vínculo que son capaces de establecer entre estudiantes de distintas disciplinas, además del beneficio de interrelacionarse profesionalmente para el enriquecimiento y el desarrollo del juego y, por tanto, de la asignatura. Hay que acentuar la dificultad añadida que tiene para el profesor, ya que no hay un desarrollo del juego igual a otro porque el rumbo que toma la historia no está determinado y dependerá de las decisiones de los jugadores, siendo una aventura diferente en cada juego que cobre vida. También la labor del profesor y la forma de plantear la actividad son evaluables por parte de los alumnos, a fin de servir de mejora y perfeccionamiento para futuras representaciones del juego. El medio o el momento utilizado para la evaluación han de adaptarse a lo que se quiere evaluar: puede ser a través de informe, cuestionario, portafolio o exposición oral de la experiencia.

Variable 7. Alumnos sin rol

En caso de que el grupo inicial del que parte el docente sea demasiado grande, será inviable que todos los alumnos puedan participar en un solo juego. Se podrán organizar diferentes subgrupos o sesiones, para que participen todos, o simplemente diseñar la actividad como de carácter voluntario. En ambos casos, habrá que prever qué hacen los alumnos sin rol. A los alumnos que quedan fuera de la representación se les puede asignar ocupaciones que sirvan de apoyo al jugador, se les puede pedir que realicen críticas constructivas a la simulación que llevan a cabo sus compañeros, o que la evalúen, todo ello a fin de darles la oportunidad de beneficiarse de la actividad, no sólo desde fuera del juego, sino también animándoles a que sus aportaciones sirvan de utilidad a los compañeros que están dentro del juego.

Variable 8. Motivación para los estudiantes.

El propio juego es un estímulo para el alumno que tiene que meterse en la piel de un personaje y se enfrenta junto con sus compañeros a la resolución de un problema, que es el propósito del juego. Al encarnar al personaje, el alumno, tratará por todos los medios de ir conociendo los datos necesarios para llegar a la solución. La utilidad de conocer la información que necesita para avanzar se pone de manifiesto y es objeto directo de interés para el jugador, con lo que, de forma natural, el contenido de la asignatura atrae toda la atención del alumno, que tratará de conocerla bien y de forma voluntaria para seguir disfrutando del juego, animándose a contraer la responsabilidad necesaria para afrontar decisiones. Además de la motivación con que cuentan tradicionalmente los estudiantes (la evaluación y su correspondiente calificación), también pueden obtener créditos de libre configuración, certificados de aprovechamiento o puntos extra, sin

Deborah García Magna, Cristina Castillo Rodríguez, Sonia Rios

$406 \quad \begin{gathered}\text { Moyano, Carmen Cristofol Rodríguez, Ma Jesús Carrasco } \\ \begin{array}{c}\text { Santos, Rosa Ma } M^{a} \text { Rodríguez Mérida, Inmaculada Pastor García y } \\ \text { David González Ramírez }\end{array}\end{gathered}$




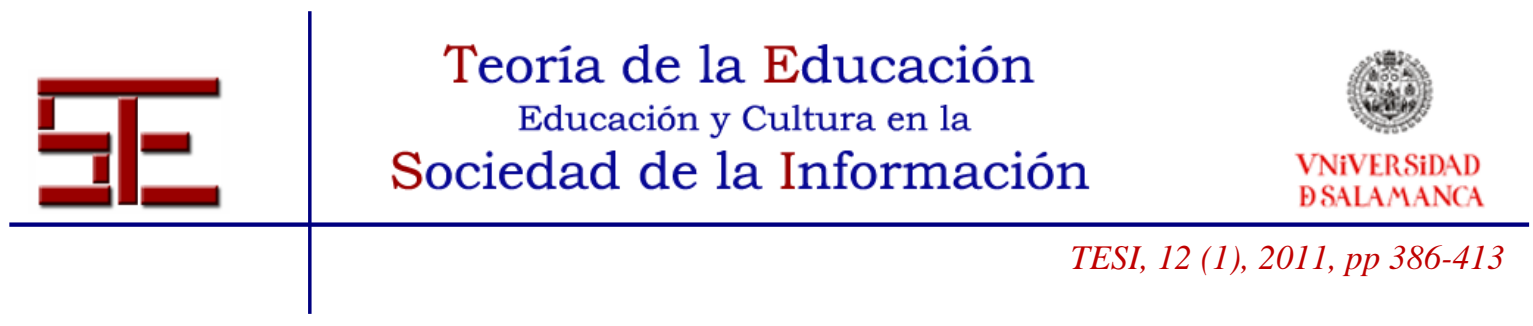

descartar premios adicionales que se puedan ofrecer por una participación exitosa en el juego.

Variable 9. Retroalimentación para el docente.

Será conveniente tras experimentar el juego de rol, comprobar la ventaja educativa que nos aporta, y si el esfuerzo que requiere la preparación que precede al juego compensa los resultados alcanzados. En caso de ver clara la ventaja comparativa con otros métodos de enseñanza, hay que indagar en aquellas particularidades que sean mejorables para futuras representaciones. Se trata de analizar cómo valoran los alumnos el juego realizado, si se incrementa la asistencia a clase, si hay mejora en el nivel y calificaciones obtenidas por los alumnos, etc., por ejemplo, mediante entrevistas personales y debates que clarifiquen y detallen aspectos mejorables en el desarrollo del juego, no sólo con los estudiantes sino también a través del diálogo entre los profesores de distintas disciplinas que estén participando en un juego común. Se trata de observar además las distintas reacciones que ha tenido el alumno ante los diferentes escenarios que se han presentado en el juego, comprobar si estaban o no previstas por el profesor, y planificar nuevas reacciones. También hay que analizar las posibilidades de la asignatura y del tiempo disponible para realizar nuevos juegos dentro del mismo curso.

Variable 10. Recursos humanos y materiales.

Otro aspecto a tener en cuenta son las facilidades que se dan al alumnado para la realización del juego, aportando tecnologías de la información y la comunicación, elementos que le ayuden a entrar en el papel (como fichas descriptivas del personaje o vestuario apropiado al contexto), y libros y material de consulta abundante para obtener información necesaria que apoye la realización de la actividad. Además del material físico se pueden aportar recursos humanos que ayuden a dar vida al juego, añadiendo otro personal de apoyo que colabore en el desarrollo fluido y eficaz del mismo, en caso de ser solicitado por el alumno. Dicho personal estará asesorado para aconsejar al estudiante (se menciona como ejemplo el personal de biblioteca o técnicos informáticos, pero el personal de apoyo puede ser tan amplio y versátil como los distintos campos de conocimiento en los que nos encontremos).

Una vez presentada la guía para el diseño de juegos de rol interdisciplinares, y aunque ya se han apuntado a lo largo de este texto algunas de las ventajas de su aplicación en las aulas, no queremos dejar de señalar de manera pormenorizada cómo esta metodología contribuye a que los estudiantes adquieran competencias fundamentales

Deborah García Magna, Cristina Castillo Rodríguez, Sonia

Rios Moyano, Carmen Cristofol Rodríguez, Ma Jesús

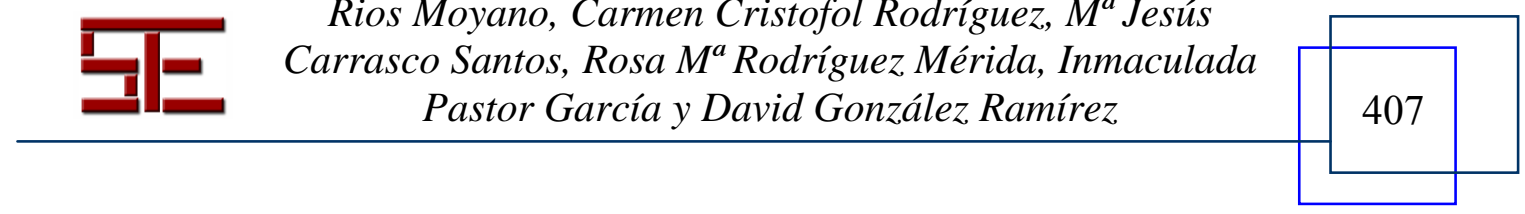




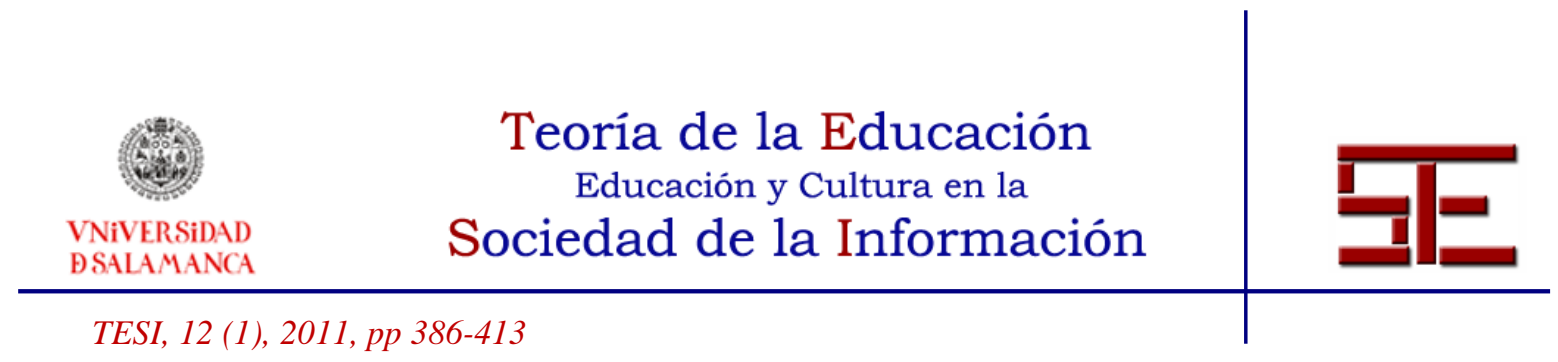

para su futuro profesional y ayuda a que los profesores mejoren sus habilidades docentes.

\section{5.- APORTACIONES DIDÁCTICAS DEL JUEGO DE ROL INTERDISCIPLINAR}

El juego de rol interdisciplinar como metodología de aprendizaje supone un aporte sustantivo en la labor que el docente desarrolla dentro del nuevo sistema educativo. Las razones son de diverso grado y alcance, aunque apriorísticamente podríamos establecer la comunidad del profesorado y el colectivo del alumnado como centros de gravedad en los que nuestra innovación supone un cambio transformatorio.

\section{1.- $\quad$ El profesorado: un ejercicio de renovación educativa}

En el ámbito docente, y teniendo en consideración que tratamos de imprimirle al tradicional juego de rol un enfoque novedoso desde la conjugación de varias disciplinas, este modelo lleva aparejado todo un reto en el afán de superación como docentes, principalmente por el intento de congregar distintas áreas de conocimiento que cuentan en su base formativa con una serie de elementos teóricos, habilidades prácticas, competencias y valores.

La contribución que esta metodología educativa tiene para el profesor se podría explicar desde estos cuatro núcleos:

1. Reciprocidad de comunicación

2. Transferencia de conocimiento

3. Investigación educativa

4. Interactividad curricular

La reciprocidad en la comunicación entre las distintas disciplinas a las que se puede aplicar dicho modelo supone un indudable enriquecimiento para el profesorado, pues éste puede observar y valorar cómo el campo de acción de su área de conocimiento se expande y ramifica hacia nuevas fórmulas curriculares, acercando de forma interactiva una realidad social y cultural cada vez más próxima al alumno en su futura integración en el ámbito laboral.

La transferencia de conocimiento en este modelo de aprendizaje comunicativo implica a la vez la transmisión del mismo de forma circular y la posibilidad de acceder a otro tipo

Deborah García Magna, Cristina Castillo Rodríguez, Sonia Rios

$408 \quad \begin{gathered}\text { Moyano, Carmen Cristofol Rodríguez, Ma Jesús Carrasco } \\ \begin{array}{c}\text { Santos, Rosa Ma Rodríguez Mérida, Inmaculada Pastor García y } \\ \text { David González Ramírez }\end{array}\end{gathered}$




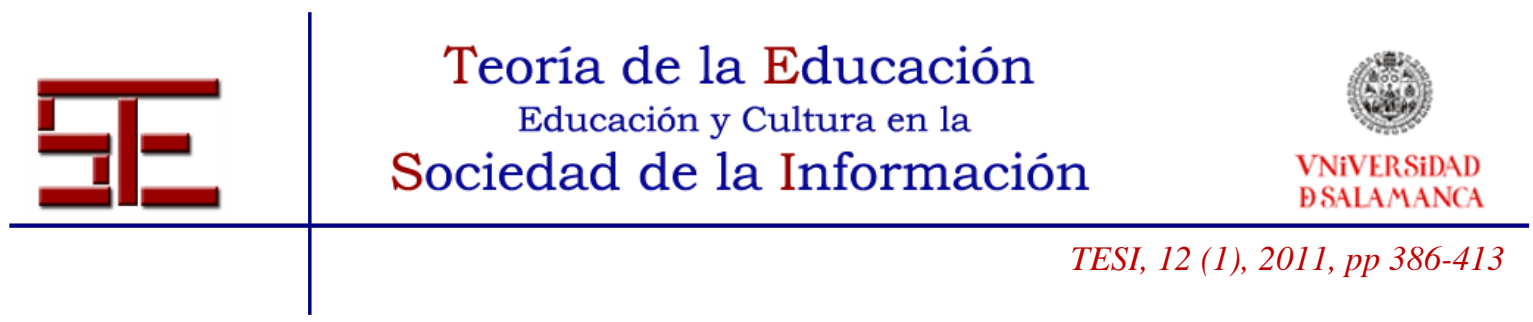

de valores, más universales y menos específicos, donde prevalezca la investigación educativa.

El profesor deberá distanciarse de sus métodos tradicionalistas y ampararse en modelos novedosos de educación, trabajando para ello con fórmulas nunca antes avistadas en su programa educativo. Por tanto, la investigación ahora no se verá reducida a un único nivel, el del área de conocimiento, sino que se desarrollará en un nuevo campo de acción, el de la educación.

El intercambio de juicios, experiencias y modelos educativos con expertos de otras disciplinas, en las que varía desde el número de alumnos hasta el papel que éstos desempeñarán en el futuro, será también una contribución notable para los docentes que intervengan en esta metodología interdisciplinar.

\section{2.- $\quad$ El alumnado: ventajas interdisciplinares}

Desde el punto de vista del alumno, consideramos que la flexibilidad que ofrece este tipo de aprendizaje conlleva una interesante vía para acceder a nuevas experiencias educativas, desarrolladas en escenarios distintos y en los que se comparten contenidos disímiles mediante un aprendizaje abierto a la cooperación, y para participar como miembros activos de una comunidad universitaria, en la que se tratará de aportar conocimientos adquiridos y resolver cuestiones que atañen a su disciplina en particular, sin desatender nunca las contribuciones venidas desde otras laderas del conocimiento.

También podríamos resumir en cuatro las aportaciones de las que el alumno se puede beneficiar:

1. Incentivar la creatividad y la experimentación

2. Fomentar la capacidad de diálogo

3. Aprender a trabajar en grupo

4. Aplicar los conocimientos adquiridos

Creemos que destacar la creatividad en la educación supone para el alumno la apreciación y receptividad respecto de las ideas nuevas y la curiosidad en la experimentación de problemas. De esta manera el alumno podrá fomentar la inventiva y desarrollar el espíritu innovador, dos bazas fundamentales muy demandadas en una sociedad de competencias como la del siglo XXI.

Deborah García Magna, Cristina Castillo Rodríguez, Sonia

Rios Moyano, Carmen Cristofol Rodríguez, Mesús

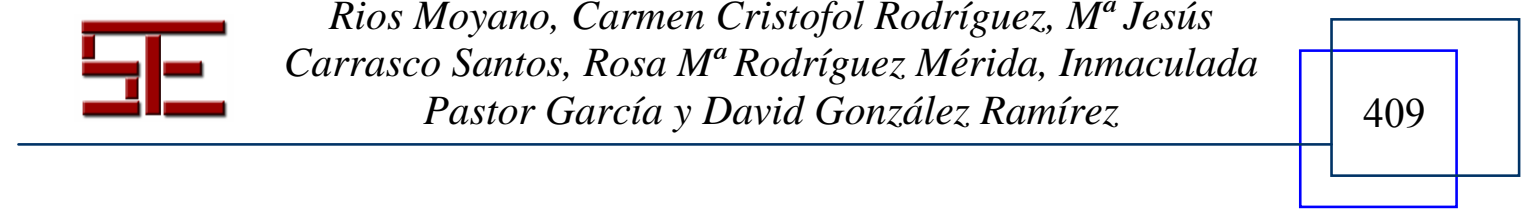




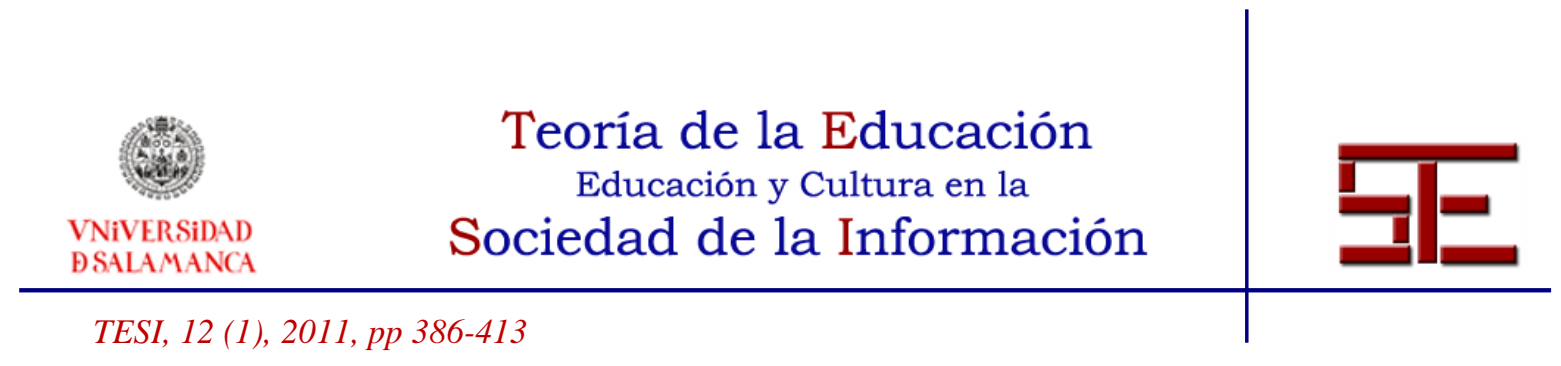

El estudiante no puede ya seguir participando de esa inactividad, de esa pasividad, a la que ha estado relegado durante décadas. Tiene que reivindicar su figura como alumno y sentirse protagonista de su propia educación. En otro tipo de sociedades, sería inconcebible pensar que el alumno es un ser del cual destaca por encima de todo su inoperancia en el proceso enseñanza aprendizaje. Hay que volver los ojos a los orígenes del conocimiento, a aquellas sociedades en las que el alumno adquiría sabiduría porque tenía curiosidad por conocer y aprender.

No se puede, en muchos casos en los que no es funcional, seguir perpetuando el trabajo individual; conocemos ya las ventajas de la colaboración grupal, a través de la cual el alumnado recibe aportaciones experienciales y académicas. La sociedad no se concibe sin el engranaje de un grupo de trabajo (y dadas las rivalidades del mercado, cada vez son más las competencias que debe reunir un especialista en una materia determinada). Por tanto, es imprescindible que en el aula se comience a trabajar en grupo para que cada alumno alcance valores que de forma individual sería imposible que desarrollase.

Con una prueba escrita a final de curso difícilmente llegará a calibrarse el nivel de conocimiento adquirido por el alumno. Pero mucho más complicado será valorar otra serie de competencias que el alumnado deberá de haber alcanzado. Con la aplicación de conocimientos que el juego de rol exige, estamos seguros de que el alumno sabrá manejarse con más soltura en los límites de sus disciplinas, e igualmente no dudamos de que él mismo se convencerá de que circunscribiéndose a la serie de apuntes dictados por el profesor y documentándose con varios manuales de asedio común, no podrá hacerse un hueco en un mundo laboral que demanda cada vez más la polivalencia de los universitarios, su capacidad interdisciplinar.

\section{6.- CONCLUSIONES}

En la actualidad presenciamos una sociedad cada vez más compleja donde surgen problemas que deben ser abordados desde múltiples perspectivas para encontrar diferentes tipos de soluciones rentables. Asimismo, hoy en día, a los profesionales se les exige una formación cada vez más polivalente, disponibilidad de habilidades transversales (en especial, capacidad de trabajo colaborativo), el reconocimiento de nuevos roles y la capacidad de adaptación a una sociedad que está en continuo cambio.

Este planteamiento del mundo actual hace que sea necesaria una profunda imbricación entre distintas áreas de conocimiento ya desde el propio título de grado. Una de las técnicas docentes que más se ajusta a esta realidad es el denominado julego de rol”, ya

Deborah García Magna, Cristina Castillo Rodríguez, Sonia Rios

$410 \quad \begin{gathered}\text { Moyano, Carmen Cristofol Rodríguez, Ma Jesús Carrasco } \\ \begin{array}{c}\text { Santos, Rosa Ma } M^{a} \text { Rodríguez Mérida, Inmaculada Pastor García y } \\ \text { David González Ramírez }\end{array}\end{gathered}$




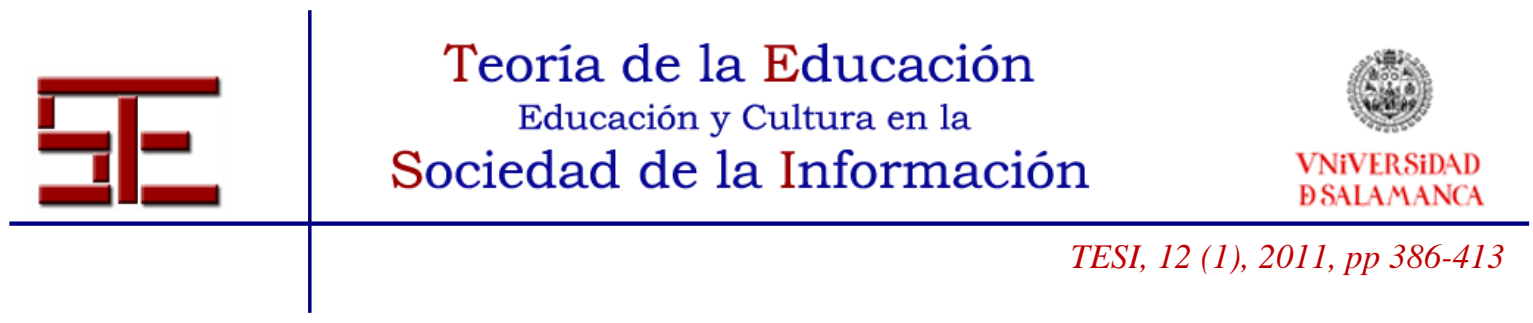

que contribuye sobremanera a la mejora del proceso de enseñanza aprendizaje y permite, mejor que cualquier otra metodología, la simulación de situaciones profesionales con implicaciones multidisciplinares.

No obstante, existen muy pocos estudios empíricos sobre el juego de rol en educación superior. Deducimos que una de las razones por las cuales esta técnica no se ha incorporado de manera natural a la práctica docente y, por ende, no se ha investigado en profundidad, estriba en que no existen pautas generales para la organización, diseño e implementación de juegos de rol en el ámbito universitario. Se trata de una técnica dinámica y compleja, con multitud de variables a tener en cuenta, que requiere por ello una gran inversión de tiempo y esfuerzo para el docente que desea ponerla en práctica por primera vez. Esta carencia se pone especialmente de manifiesto si añadimos el componente interdisciplinar que es, a nuestro juicio, la gran baza de esta metodología.

Por ello, hemos iniciado un proyecto de investigación con el objetivo de facilitar la puesta en práctica de los juegos de rol interdisciplinares, partiendo de nuestra propia experiencia como profesores de diversas áreas de conocimiento. Proponemos en este artículo una guía que sirva de modelo al docente que quiera beneficiarse de las ventajas de esta metodología, definiendo de forma clara los pasos a seguir y las variables a tener en cuenta a la hora de crear y poner en práctica un juego de rol interdisciplinar.

\section{7.- $\quad$ BIBLIOGRAFÍA}

AAVV. (2005). Actividades para la enseñanza y el aprendizaje de competencias genéricas en el marco del Espacio de Educación Europeo Superior. Zaragoza: Prensas Universitarias.

Armstrong, E. K. (2003). Applications of Role Playing in Tourism Management teaching: Evaluation of a Learning Method. Journal of Hospitality, Leisure, Sport and Tourism Education. 2 (1), 5-16.

Bricall, J. (2000). Informe Universidad 2000. Barcelona, España, marzo de 2000. Extraído el 30 Agosto, 2010, de http://www.oei.es/oeivirt/bricall.htm

Corpas Pastor, G.; Amaya Galván, M. C.; Bautista Zambrana, M.R.; Castillo Rodríguez, C.; Leiva Rojo, J.; Seghiri Domínguez, M.; Serrano Alcaide, C.; Zambrana Moral, P. (2005). Simulación de la gestión empresarial de proyectos de traducción científico-técnica a través de una plataforma DEV. En Innovación Educativa y Enseñanza Virtual de la Universidad de Málaga (pp-1-13). Málaga:

Deborah García Magna, Cristina Castillo Rodríguez, Sonia

Rios Moyano, Carmen Cristofol Rodríguez, Mesús

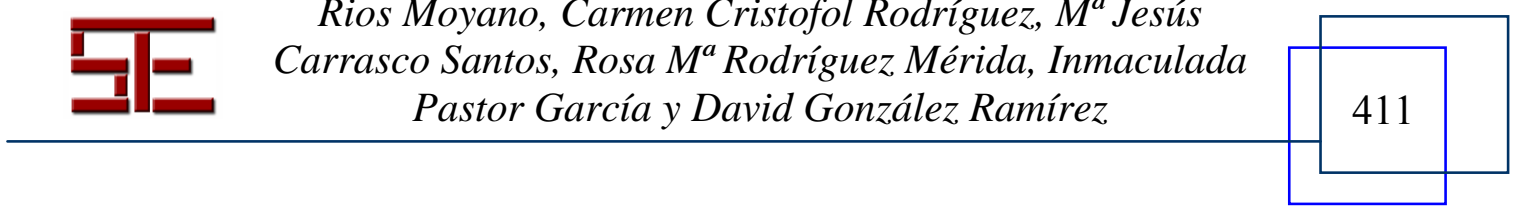




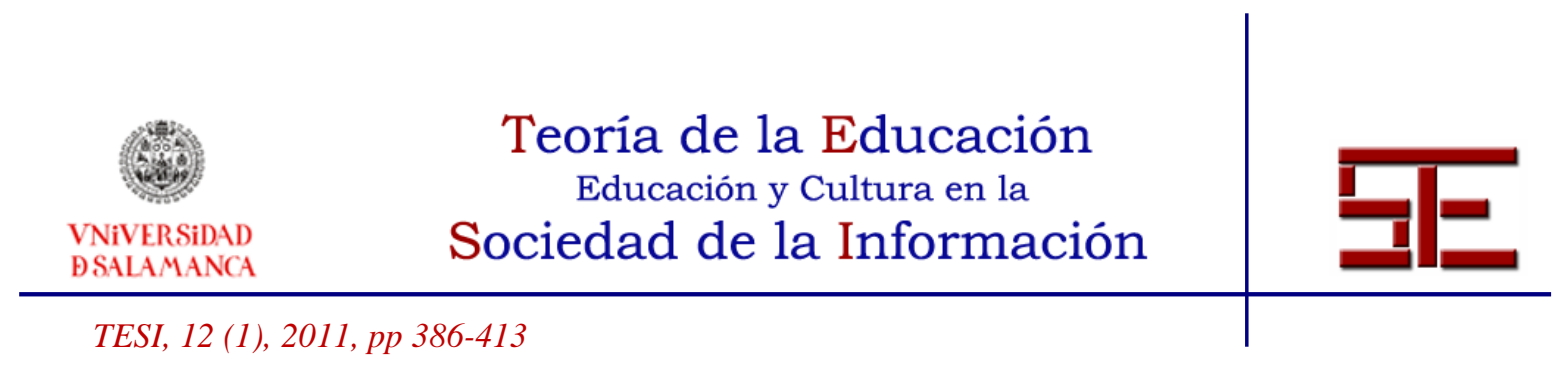

Servicio de Innovación Educativa y Servicio de Enseñanza Virtual y Laboratorios Tecnológicos de la Universidad de Málaga.

Delors, J. (coord.). (1996). La educación encierra un tesoro. Informe a la UNESCO de la Comisión Internacional para la Educación del siglo XXI. Ediciones UNESCO, Santillana, 1996. Extraído el 30 Agosto, 2010, de http://www.unesco.org/education/pdf/DELORS_S.PDF

Feinstein, A. H., Mann, S., \& Corsun, D.L. (2002). Charing the Experiential Territory: Clarifying Definitions and Uses of Computer Simulation Games and Role Play. Journal of Management Development. 21 (10), 732-744.

Gil Flores, J.; Álvarez Rojo, V.; García Jiménez, E.; Romero Rodríguez, S. (2004). La enseñanza universitaria, planificación y desarrollo de la docencia. Madrid: EOS.

Hernández Díaz, A. (2000). Teoría crítica de la enseñanza. En CEPES, Tendencias pedagógicas en la realidad educativa actual (pp. 115-125). Tarija-Bolivia: Editorial universitaria Universidad Juan Misael Saracho. Extraído el 30 Agosto, 2010, de http://cvi.mes.edu.cu/gucid/Members/mtperez/978959160676101.pdf

Hsu, E. (1989). Role Event Gaming Simulation in Management Education: A Conceptual Framework and Review. Simulation and Games. 20 (4), 409-438.

Japiassu, H. (1976). Interdisciplinaridade e patologia do saber. Rio de Janeiro: Imago.

Jiménez Martín, S. y Moncholi Chaparro, M.A. (2009). El entrenamiento en técnicas creativas en el Espacio Europeo de Educación Superior. En Actas del I Congreso Internacional Latina de Comunicación Social (pp. 1-12). Tenerife: Universidad de La Laguna. http://www.revistalatinacs.org/09/Sociedad/actas/12silvia.pdf

Ladousse, G.P. (1987). Role Play. Oxford: Oxford University Press.

Lean, J., Moizer, J., Twoler, M. \& Abbey, C. (2006). Simulations and Games: Use and Barriers in Higher Education. Active Learning in Higher Education. 7, $227-242$.

León Vegas, M. (2009, octubre). El aprendizaje autónomo a partir del juego de rol en la disciplina de historia moderna. Ponencia presentada en el Congreso Internacional de Innovación Presente y futuro en la Docencia Universitaria, Huelva, España.

Morin, E. (1994). Introducción al pensamiento complejo. Barcelona: Gedisa.

Morin, E. (2001). La mente bien ordenada: repensar la reforma, reformar el pensamiento. Barcelona: Seix Barral.

Moss, B. (2000). The Use of Large-Group Role-Play Techniques in Social Work Education. Social Work Education. 19 (5), 471-483.

Oberle, A. P. (2004). Understanding Public Land Management through Role Play". Journal of Geography. 103 (5). 199-210.

Ortega y Gasset, J. (1930). Misión de la universidad. Madrid: Revista de Occidente.

Deborah García Magna, Cristina Castillo Rodríguez, Sonia Rios

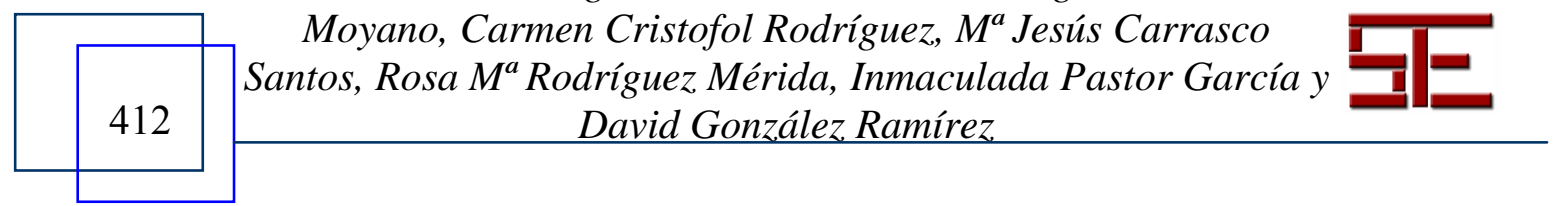




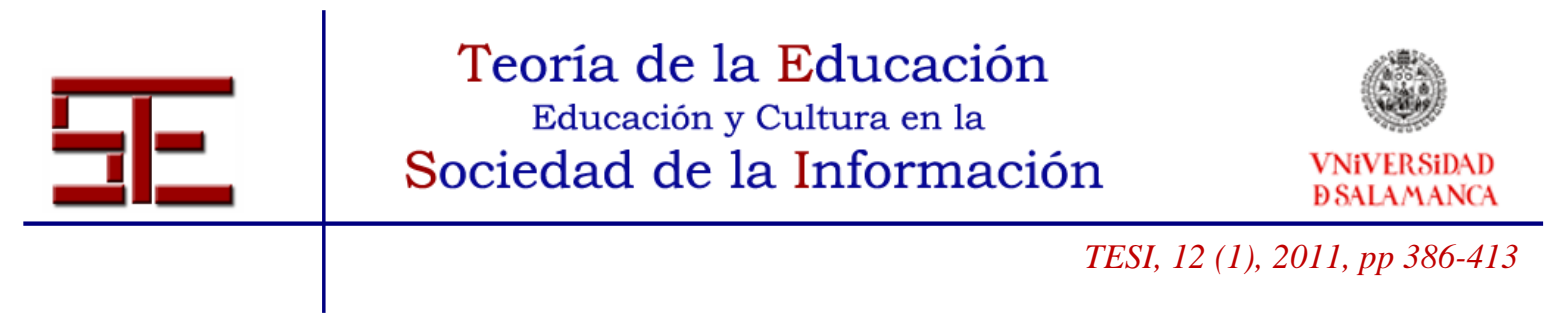

Pérez i Garcias, A. (2002). Nuevas estrategias didácticas en entornos digitales para la enseñanza superior. En J. Salinas y A. Batista (Eds.), Didáctica y Tecnología Educativa para una comunidad en un mundo digital (pp. 1-26). Panamá: Universidad de Panamá, Facultad de Ciencias de la Educación.

Piaget, J. (1979). La epistemología de las relaciones interdisciplinarias. En L. Apostel, G. Bergerr, A. Briggs \& G. Michaud (Eds.) Problemas de la enseñanza y de la investigación en las universidades (pp. 153-171). México: Asociación Nacional de Universidades e Institutos de Enseñanza Superior.

Posada Álvarez, R. (2004). Formación superior basada en competencias, interdisciplinariedad y trabajo autónomo del estudiante. Revista Iberoamericana de Educación. Sección De los lectores-Educación Superior. Extraído el 30 Agosto, 2010, de http://www.rieoei.org/deloslectores/648Posada.PDF

Ruben, B.D. (1999). Simulations, Games, and Experience Based Learning: The Quest for a New Paradigm for Teaching and Learning. Simulation and Gaming. 30 (4), 498-505.

Senge, P.M. (1996). La quinta disciplina. El arte y la práctica de la organización abierta al aprendizaje. Barcelona: Granica.

Sleigh, C. (2004). Using Role Play as a way into the History of Science. Discourse Learning and Teaching in Philosophical and Religious Studies. 3 (2), 131-141.

\footnotetext{
${ }^{1}$ En nuestro país el crédito LRU equivalía a 10 horas de docencia impartidas.

${ }^{2}$ Conocido también comúnmente con el nombre de Informe Bricall. Para consultar el contenido íntegro de dicho informe remitimos a la siguiente URL donde se encuentra albergado: http://www.oei.es/oeivirt/bricall.htm.

3 Remitimos a la publicación contenida en Corpas et al. (2005), donde se emplea la técnica de la simulación para representar determinados aspectos de la gestión empresarial en el ámbito de la traducción.

${ }^{4}$ Iniciada a principios del siglo XX en el Instituto de Investigación Social de Frankfurt
}

Para citar el presente artículo puede utilizar la siguiente referencia:

García Magna, D., Castillo Rodríguez, C., Rios Moyano, S., Cristofol Rodríguez, C., Carrasco Santos, M. J., Rodríguez Mérida, R. M., Pastor García, I. y González Ramírez, D. (2011). La interdisciplinariedad en la educación superior: propuesta de una guía para el diseño de juegos de rol, en Hernández Serrano, M. J y Fuentes Agustí, M. (Coords.) La red como recurso de información en educación. Revista Teoría de la Educación: Educación y Cultura en la Sociedad de la Información. Vol. 12, $\mathrm{n}^{\mathrm{o}} 1$. Universidad de Salamanca, pp. 386-413 [Fecha de consulta: dd/mm/aaaa].

http://campus.usal.es/ revistas_trabajo/index.php/revistatesi/article/view/7894/7937

Deborah García Magna, Cristina Castillo Rodríguez, Sonia

Rios Moyano, Carmen Cristofol Rodríguez, Ma Jesús

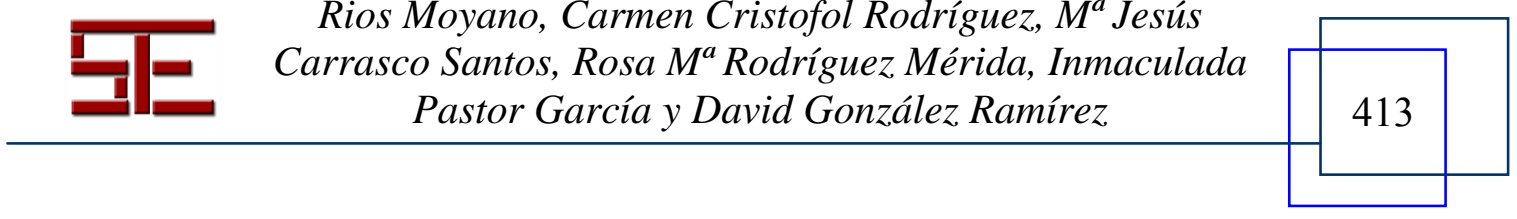

